\title{
Potential contribution of SIM2 and ETS2 functional polymorphisms in Down syndrome associated malignancies
}

Arpita Chatterjee ${ }^{1}$, Samikshan Dutta 1,6, Sanjit Mukherjee ${ }^{2}$, Nupur Mukherjee ${ }^{3}$, Avirup Dutta ${ }^{2}$, Ashis Mukherjee ${ }^{4}$ Swagata Sinha ${ }^{1}$, Chinmay Kumar Panda ${ }^{3}$, Keya Chaudhuri ${ }^{2}$, Ananda L Roy ${ }^{5}$ and Kanchan Mukhopadhyay ${ }^{1 *}$

\begin{abstract}
Background: Proper expression and functioning of transcription factors (TFs) are essential for regulation of different traits and thus could be crucial for the development of complex diseases. Subjects with Down syndrome (DS) have a higher incidence of acute lymphoblastic leukemia (ALL) while solid tumors, like breast cancer (BC) and oral cancer (OC), show rare incidences. Triplication of the human chromosome 21 in DS is associated with altered genetic dosage of different TFs. V-ets erythroblastosis virus E26 oncogene homolog 2 (ETS2) and Single Minded 2 (SIM2) are two such TFs that regulate several downstream genes involved in developmental and neurological pathways. Here we studied functional genetic polymorphisms (fSNP) in ETS2 and SIM2 encoding genes in a group of patients and control subjects to better understand association of these variants with DS phenotypes.
\end{abstract}

Methods: We employed an in silico approach to identify potential target pathways of ETS2 and SIM2. fSNPs in genes encoding for these two TFs were identified using available databases. Selected sites were genotyped in individuals with DS, their parents, ALL, BC, OC as well as ethnically matched control individuals. We further analyzed these data by population-based statistical methods.

Results: Allelic/genotypic association analysis showed significant $(P<0.03)$ differences of rs2070530, rs1051476, rs 11254, rs711 for DS subjects compared to control. rs711 also exhibited significantly different genotypic distribution pattern in parents of DS probands $(P<0.02)$ and $B C$ patients $(P<0.02)$. Interaction analysis revealed independent main effect of rs711 in all the groups, while rs 11254 exhibited independent main effect in DS subjects only. High entropy values were noticed for rs461155 in the solid tumor groups. Significant interactive effects of rs2070531 with rs1051475, rs1051476, rs11254 were observed in all the groups except DS.

Conclusions: We infer from the present investigation that the difference in frequencies of fSNPs and their independent as well as interactive effects may be the cause for altered expression of SIM2 and ETS2 in DS and malignant groups, which affects different downstream biological pathways. Thus, altered expression of SIM2 and ETS2 could be one of the reasons for variable occurrence of different malignant conditions in DS.

Keywords: SIM2, ETS2, Down syndrome, Breast cancer, Oral cancer, Acute lymphoblastic leukemia

\footnotetext{
* Correspondence: kanchanmvk@yahoo.com

${ }^{1}$ Manovikas Biomedical Research and Diagnostic Centre, MRIH, 482,

Madudah, Plot I-24, Sec.-J, E.M. Bypass, Kolkata 700107, India

Full list of author information is available at the end of the article
} 


\section{Background}

Transcription factors (TFs) regulate pathways related to diseases either through their direct action on the target genes or by controlling downstream pathways. Hence they are important candidates for investigating etiology of complex diseases. There are several TF encoding genes in the human $21^{\text {st }}$ chromosome (HSA21) and deregulated expression of any of these could influence downstream pathways. Due to trisomy of the HSA21 in Down syndrome (DS) (MIM\# 190685), genetic overdosage of a number of TF encoding genes is a distinct possibility. DS patients are prone to acute leukemia, including acute lymphoblastic leukemia (ALL), while solid tumors especially breast cancer $(\mathrm{BC})$ is rare [1]. We hypothesized that DS related abnormalities like intellectual disability, immunological imbalance, hormonal alteration, and predisposition to childhood acute leukemia could be due to improper expression and functioning of TFs located in the HSA21. Because disease association studies have revealed higher differential expression ratio in different tissues for the TF genes encoding Single minded 2 (SIM2) and V-ets erythroblastosis virus E26 oncogene homolog 2 (ETS2) within HSA21 [2], here we explored the role of these two TFs in DS phenotype and related malignancies.

SIM2 is important for normal neuronal development. SIM2 can heterodimerize with aryl hydrocarbon receptor nuclear translocator (ARNT) and translocate to the nucleus to transcriptionally regulate gene expression [3]. Expression of SIM2 mRNA has been detected in fetal brain regions associated with DS pathology [4]. SIM2 also plays an important role in carcinogenesis. After entry into a cell, carcinogenic compounds bind to the cytoplasmic Aryl hydrocarbon receptor (AhR) and are carried to the nucleus. Ligand-bound AhR together with ARNT [5] bind to the Xenobiotic Response Element present in the promoter region of certain genes encoding for oxidative enzymes [6-8]; transcriptional activation of these enzymes accelerates carcinogen metabolism [9]. SIM2 inhibits AhR/ARNT dimerization, thereby inhibiting carcinogen metabolism and promoting carcinogenesis [5,10]. In addition, SIM2 is the second most consistently over expressed gene in prostate cancer [11] and over expression of the short isoform of SIM2 (SIM2s) is reported in malignant colon, pancreas, and prostate tissues as compared to the corresponding normal tissues [9-11]. SIM2 has further been proposed to have a breast tumor suppressive activity [12] and a genome-wide linkage scan identified three putative breast cancer susceptibility loci, one of which (21q22) harbors SIM2 [13]. Therefore, SIM2 functions as a tumour selective marker and drug target in several types of malignancies [10].

Besides SIM2, ETS2 over expression induces craniofacial defects as well as skeletal anomalies in transgenic mice resembling DS [14]. Increased rate of neuronal apoptosis [15] and amyloid precursor protein (APP) gene transactivation are also observed upon ETS2 over expression [16], which might play an important role in the early onset of Alzheimer's disease and neuronal abnormalities in DS [16].

ETS2 can act both as a transcriptional activator as well as a repressor during cellular proliferation, differentiation and tumorigenesis [17-26]. For instance, cell cycle regulator genes like bcl-xL, c-myc, cyclin D1 and p53 are activated by ETS2 [27,28], while BRCA1 expression is repressed in breast cancer tissue [29]. Interestingly, certain genetic translocations in ETS2 were observed in DS patients suffering from leukemia [30]. An interaction of ETS2 and ERG with GATA1 mutations were reported in DS subjects with acute megakaryoblastic leukemia and activation of the JAK/STAT pathway, a frequent attribute of megakaryocytic malignancies, was identified as common phenomena for this malignant transformation [31].

Given the functional importance of SIM2 and ETS2, we sought out to investigate alterations in their expression in disease etiology. Functional single nucleotide polymorphisms (fSNP) in candidate genes are important indicators for their association with disease phenotypes. In the present study, fSNPs of SIM2 and ETS2 were analyzed for their potential role in Indian individuals suffering from DS, ALL and solid tumors that includes BC and oral cancer $(\mathrm{OC})$.

\section{Methods}

\section{In Silico analysis to predict pathways regulated by SIM2 and ETS2}

We undertook computational methods to determine the probable pathways regulated by SIM2 and ETS2. The promoter sequences (from-5000 bp to $+1000 \mathrm{bp}$ ) were retrieved from the Eukaryotic promoter database (EPD) (http://www.epd.isb-sib.ch/) and Transcriptional Regulatory Element Database (TRED) (http://rulai.cshl.edu/ TRED). Presence of SIM2 and/or ETS2 binding sites in the promoter sequences were identified by a Perl based program "Consensus-Finder".

We retrieved expression profile of the genes harboring binding sites for SIM2 and ETS2 in different tissues by using GNF SymAtlas database (http://symatlas.gnf.org/ SymAtlas/). Tissue-specific differential expression pattern (fold change) were calculated separately by comparing the median value of expression; value greater than the median was considered as over expression and value less than median was considered as under expression. We then determined co-expression of putative target genes at the site of over expression/under expression of SIM2 and ETS2 and promoter sites of these genes were analyzed by GENEDOC and Promoter Scan tools respectively (http://www-bimas. cit.nih.gov/molbio/proscan/). Functions of these putative 
target genes along with SIM2 and ETS2 in various biological pathways was analyzed by Panther (http://www. pantherdb.org/pathway/) and KEGG pathway (http://www. genome.ad.jp/kegg/pathway.html). The entire process is presented schematically in Figure 1.

\section{In silico identification of functional variants}

We used different web based tools namely SIFT (http:// sift.jcvi.org), PolyPhen (http://coot.embl.de/PolyPhen/), SNPs3D (http://www.snps3d.org/), Pupasuite 2 (http:// pupasuite.bioinfo.cipf.es), GlobPlot, FastSNP (http://fastsnp. ibms.sinica.edu.tw), SNP@Promoter (http://variome.kobic. re.kr/SNPatPromoter), and dbSMR (http://miracle.igib.res. in/dbSMR) to identify fSNPs in the genomic regions of SIM2 and ETS2 as reported earlier [32]. fSNPs selected for genotyping in the present study are listed in Table 1.

\section{Subjects}

Five ethnically matched groups of individuals were recruited for analysis of fSNPs. Healthy volunteers, without any clinical history of intellectual disability or malignant disorder, were recruited as controls $(\mathrm{N}=149)$. Nuclear families having child with DS $(\mathrm{N}=132)$ were recruited from the outpatient department of Manovikas Kendra, Kolkata and trisomic status of the probands was confirmed by karyotyping. ALL patients $(\mathrm{N}=38)$ were recruited from the Netaji Subhash Chandra Bose Cancer Research Institute, Kolkata. Genomic DNA from post-operative normal tissue, adjacent to malignant $\mathrm{BC}(\mathrm{N}=49)$ and $\mathrm{OC}(\mathrm{N}=54)$ were collected from Chittaranjan National Cancer Research Institute and Indian Institute of Chemical Biology, Kolkata respectively. All samples were acquired after obtaining

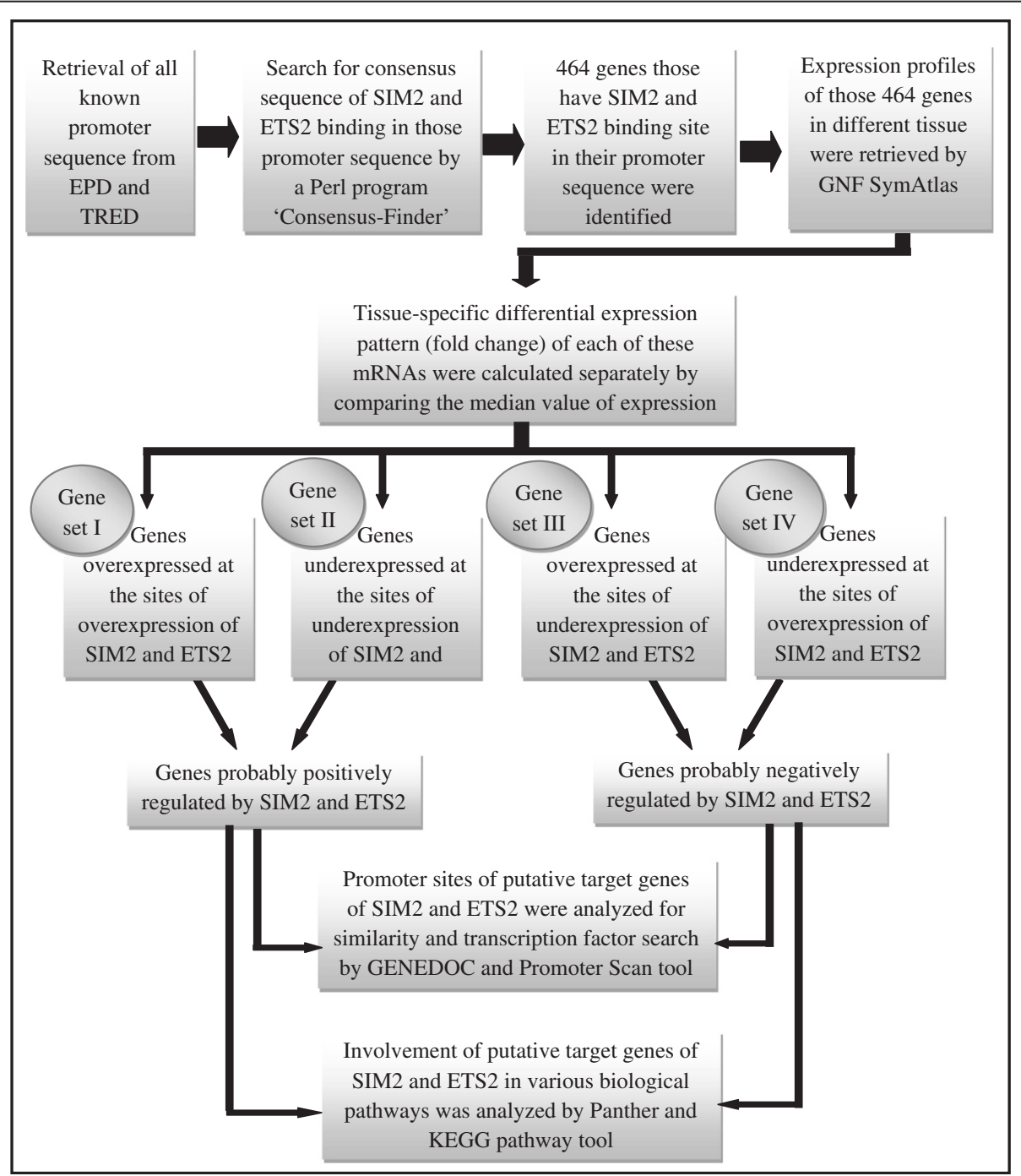

Figure 1 Schematic presentation of the in silico methods used for identification of putative target pathways of SIM2 and ETS2. 
Table 1 Details on SIM2 and ETS2 functional SNPs explored in this study

\begin{tabular}{|c|c|c|c|c|c|}
\hline Gene & SNP ID & Position and type ${ }^{a}$ & Alleles $(A 1 / A 2)^{b}$ & Probable function $^{c}$ & $\begin{array}{l}\text { MAF (A2) in other populations } \\
\text { (CEU, HCB, JPT and YRI) }\end{array}$ \\
\hline \multirow[t]{7}{*}{$\overline{S I M 2}$} & rs2269188 & Intronic, regulatory & $\mathrm{G} / \mathrm{C}$ & AhR binding site & $0.305,0.567,0.534,0.092$ \\
\hline & rs2070650 & Intronic & $\mathrm{C} / \mathrm{A}$ & C-Myc binding site & $0.358,0.578,0.456,0.500$ \\
\hline & rs79727992 & Intronic & G/A & NK & 0.014 (CEU) \\
\hline & rs16994404 & Syn, Cod & $\mathrm{C} / \mathrm{T}$ & SR protein mediated splicing regulation & 100\% C (all populations) \\
\hline & rs77335240 & Intronic & $\mathrm{A} / \mathrm{C}$ & NK & No population data \\
\hline & rs78455239 & Intronic & $\mathrm{C} / \mathrm{A}$ & NK & No population data \\
\hline & rs79022672 & Intronic & $\mathrm{G} / \mathrm{A}$ & NK & 0.020 (YRI) \\
\hline \multirow[t]{28}{*}{ ETS2 } & rs34373350 & NS, cod & $\mathrm{C} / \mathrm{T}$ & Damaging & 0.006 (CEU), 0.025 (YRI) \\
\hline & rs11700777 & Syn, Cod & A/G & SR protein mediated splicing regulation & No population data \\
\hline & rs114481523 & NS, cod & $\mathbf{A} / \mathrm{G}$ & NK & $0.005(\mathrm{YRI})$ \\
\hline & rs73450556 & Intronic & $\mathbf{G} / \top$ & AML-1a banding site & 0.500 (YRI) \\
\hline & rs77688599 & Intronic & $\mathrm{G} / \mathbf{A}$ & NK & 0.056 (CEU) \\
\hline & rs60277131 & Intronic & $\mathbf{A} / \mathrm{G}$ & NK & $0.180(\mathrm{YRI})$ \\
\hline & rs78391361 & NS, cod & $\mathrm{C} / \mathrm{T}$ & NK & 0.042 (CEU) \\
\hline & rs1803557 & NS, cod & $\mathbf{A} / \mathrm{T}$ & Damaging & No population data \\
\hline & rs34472454 & FS, Cod & $-/ C$ & NK & No population data \\
\hline & rs113798497 & Syn, Cod & $\mathrm{A} / \mathrm{G}$ & NK & No population data \\
\hline & rs374575 & Intronic & $\mathrm{C} / \mathrm{T}$ & Transcriptional regulation & $0.290,0.140,0.041,0.005$ \\
\hline & rs2070529 & & $\mathrm{C} / \mathrm{T}$ & Cf1, AML-1a binding site & $0.168,0.523,0.610,0.270$ \\
\hline & rs2070530 & & $\mathbf{G} / \mathrm{C}$ & V-Myb binding site & $0.168,0.523,0.610,0.290$ \\
\hline & rs2070531 & & $\mathrm{C} / \mathrm{T}$ & NIT2 binding site change & $0.398,0.221,0.227,0.288$ \\
\hline & rs434421 & & $\mathbf{C} / \mathrm{T}$ & Nkx-2, USF binding & No population data \\
\hline & rs8128227 & & $\mathrm{C} / \mathrm{A}$ & NK & 0.014 (CEU) \\
\hline & rs6517481 & & A/G & Ttk 69 binding site & $0.361,0.318(\mathrm{HCB}+\mathrm{JPT}), 0.300$ \\
\hline & rs79863249 & & $\mathrm{C} / \mathrm{G}$ & NK & $0.011(\mathrm{HCB}+\mathrm{JPT})$ \\
\hline & rs60538921 & & A/G & Lyf-1 banding site & No population data \\
\hline & rs7276961 & & A/G & HSF binding site change & $0.347,0.466(\mathrm{HCB}+\mathrm{JPT}), 0.300$ \\
\hline & rs1051475 & $3^{\prime} U T R$ & $\mathrm{~T} / \mathrm{C}$ & Transcriptional regulation & $0.347,0.227(\mathrm{HCB}+\mathrm{JPT}), 0.200$ \\
\hline & rs1051476 & & $\mathrm{C} / \mathrm{G}$ & SR protein mediated Splicing regulation & $0.420,0.239,0.209,0.246$ \\
\hline & rs72094783 & & $-/ G A$ & NK & No population data \\
\hline & rs116542090 & & $\mathbf{G} / \mathrm{A}$ & NK & $0.008(\mathrm{YRI})$ \\
\hline & rs74551083 & & A/G & NK & No population data \\
\hline & rs11540409 & & A/G & NK & No population data \\
\hline & rs11254 & & $\mathrm{C} / \mathrm{T}$ & Alteration of miRNA target site & $0.432,0.233,0.200,0.250$ \\
\hline & rs711 & & G/A & Affects SR protein mediated ESE activity & $0.425,0.200(\mathrm{HCB}+\mathrm{JPT}), 0.441$ \\
\hline
\end{tabular}

${ }^{a} N S=$ Nonsynonymous, Syn $=$ Synonymous, Cod $=$ Coding, FS = Frame shift

${ }^{\mathbf{b}} \mathrm{A} 1=$ Major allele, $A 2=$ Minor allele, allele mentioned in bold letter is the ancestral allele.

${ }^{\mathrm{C}} \mathrm{NK}=$ Not known.

${ }^{\mathbf{d}}$ CEU: Caucasians from Utah with ancestry from western and northern Europe; YRI: Yoruba from Ibadan, Nigeria; HCB: Han Chinese from Beijing, China and JPT: Japanese from Tokyo, Japan.

informed written consent for participation. Institutional Human Ethical Committee approved the study protocol.

\section{Sample collection, DNA isolation and genotyping}

Peripheral blood ( $\sim \mathrm{ml})$ collected from control individuals, DS probands, their parents and ALL patients was used for extraction of genomic DNA [33]. Target sequences were amplified and PCR amplicons were subjected to genotyping (Table 2).

\section{Statistical analyses}

Difference in allelic and genotypic frequency of the studied fSNPs in different study groups as compared to control was calculated by simple $\mathrm{r} \times \mathrm{c}$ contingency table (http://www.physics.csbsju.edu/stats/contingency_NROW_ 
Table 2 Genotyping procedure for the studied SNPs

\begin{tabular}{|c|c|c|c|}
\hline Gene & SNP ID & Primer sequence $\left(5^{\prime}-3^{\prime}\right)$ & Genotyping procedure \\
\hline \multirow[t]{8}{*}{ SIM2 } & \multirow[t]{2}{*}{ rs2269188 } & F: CTCACCACGAGCTACCTGAA & \multirow[t]{2}{*}{ RFLP analysis of PCR product using Bsu36l } \\
\hline & & R: GACCAGGAGAGGGTTTGGTC & \\
\hline & rs2070650 & F: CAGTGCCATGGCCTITITAGA & \multirow{32}{*}{$\begin{array}{l}\text { DNA sequence analysis in ABI prism } 3130 \text { Genetic Analyzer using } \\
\text {-Big Dye sequencing kit v3.1 followed by analysis using Sequencing } \\
\text { Analysis software v 5.2. Electropherograms obtained were further } \\
\text { analyzed by Mutation Surveyor Demo V3.24 software to check for } \\
\text { new mutation }\end{array}$} \\
\hline & rs79727992 & R: CCAATACACACACAGCACCC & \\
\hline & rs16994404 & & \\
\hline & rs77335240 & & \\
\hline & rs78455239 & & \\
\hline & rs79022672 & & \\
\hline \multirow[t]{30}{*}{ ETS2 } & rs34373350 & F: GGGGGTTTCCTTCCAGACT & \\
\hline & rs11700777 & R: CTGATTGGGAAAGTCACGTGGG & \\
\hline & rs114481523 & & \\
\hline & rs73450556 & & \\
\hline & rs77688599 & & \\
\hline & rs60277131 & & \\
\hline & rs78391361 & & \\
\hline & rs1803557 & & \\
\hline & rs34472454 & & \\
\hline & rs113798497 & & \\
\hline & rs374575 & F: GTCTGATCAAGAGGCCCAAG & \\
\hline & rs2070529 & R: CAGAACCACTGGGGAATGAG & \\
\hline & rs2070530 & & \\
\hline & rs2070531 & & \\
\hline & rs434421 & & \\
\hline & rs8128227 & & \\
\hline & rs6517481 & & \\
\hline & rs79863249 & & \\
\hline & rs60538921 & & \\
\hline & rs7276961 & & \\
\hline & rs1051475 & F: TGTGTTTCTCCGACAGCTCA & \\
\hline & rs1051476 & R: TTTCATCAAGACCCCTACCG & \\
\hline & rs72094783 & & \\
\hline & rs116542090 & & \\
\hline & rs74551083 & & \\
\hline & rs11540409 & & \\
\hline & rs11254 & F: CCATTCATTCGGAGAAAACG & RFLP analysis of PCR product using Taql \\
\hline & & R: AAGGCCACGCAGCTAGTAAA & \\
\hline & rs711 & F: GCAACGGCACAGCTAATTCT & RFLP analysis of PCR product using Mspl \\
\hline & & R: AAATACAACTGTTAAGGGATTCTGA & \\
\hline
\end{tabular}

NCOLUMN_form.html). Minor allele frequency (MAF) of eleven fSNPs of Indian control individuals was also compared with four major populations studied in the HapMap project [Caucasians from Utah with ancestry from western and northern Europe (CEU), Han Chinese from Beijing, China (HCB) and Japanese from Tokyo, Japan (JPT) and Yoruba from Ibadan, Nigeria (YRI)]. Allelic odds ratios were calculated by Odds ratio calculator (http://www. hutchon.net/ConfidORnulhypo.htm). All P values obtained by allelic and genotypic association test were corrected for multiple testing by PLINK [34] and R program [http:// www.r-project.org/]. Linkage Disequilibrium (LD) between the SNPs was measured by Haploview 4.1 using default settings. Haplotype frequency of fSNPs was inspected by Unphased program (Version 2.404) [35]. Interaction among the genotypes of SIM2 and ETS2 was analyzed by multifactor dimensionality reduction (MDR) software (version 2.0 beta 8.1) [36] and values were expressed as 
Table 3 Target genes of SIM2/ETS2 and their probable function identified by in silico analysis Gene IDs Location Function (Obtained using Panther Pathway tool)

\begin{tabular}{|c|c|c|}
\hline A4GALT & $22 q 13.2$ & Carbohydrate metabolic process, lipid metabolic process, protein metabolic process \\
\hline $\mathrm{ABCB8}$ & $7 q 36.1$ & Immune system process, extracellular transport, carbohydrate metabolic process, response to toxin \\
\hline ABP1 & 7q36.1 & Oxidoreductase activity, immune system process, cellular amino acid and derivative metabolic process \\
\hline ATP1A1 & $1 \mathrm{p} 13.1$ & $\begin{array}{l}\text { Hydrolase activity, cation transmembrane transporter activity, ion channel activity, cation transport, lipid transport, lipid } \\
\text { metabolic process, cellular calcium ion homeostasis }\end{array}$ \\
\hline ATP1A4 & $1 \mathrm{q} 23.2$ & Cation transport, lipid transport, lipid metabolic process, homeostasis \\
\hline C1QL1 & $17 q 21.31$ & $\begin{array}{l}\text { Complement activation, carbohydrate transport, signal transduction, cell-cell signaling, cell adhesion, carbohydrate metabolic } \\
\text { process, lipid metabolic process, cellular component morphogenesis, mesoderm development, skeletal system development, } \\
\text { response to stimulus }\end{array}$ \\
\hline C4BPA & $1 \mathrm{q} 32.2$ & Complement activation, signal transduction, cell-cell adhesion, protein metabolic process, blood coagulation \\
\hline C4BPB & $1 \mathrm{q} 32.2$ & Complement activation, signal transduction, cell-cell adhesion, protein metabolic process, blood coagulation \\
\hline CEACAM1 & $19 q 13.2$ & Signal transduction, cell-cell adhesion \\
\hline CYB561 & $17 q 23.3$ & Oxidoreductase activity, respiratory electron transport chain \\
\hline EMCN & $4 q 24$ & Cell adhesion \\
\hline EXOSC2 & $9 q 34.12$ & Nucleobase, nucleoside, nucleotide and nucleic acid metabolic process \\
\hline FVT1 & $18 q 21.3$ & Oxidoreductase activity, metabolic process \\
\hline FXYD5 & $19 q 13.12$ & Ion channel activity, protein binding ion transport, signal transduction \\
\hline GATA3 & $10 \mathrm{p} 14$ & $\begin{array}{l}\text { Hydrolase activity, acting on ester bonds, DNA binding, transcription factor activity nucleobase, nucleoside, nucleotide and } \\
\text { nucleic acid metabolic process, endoderm development, heart development, hemopoiesis }\end{array}$ \\
\hline GNB2L1 & $5 q 35.3$ & Intracellular protein transport, signal transduction \\
\hline GTF3C5 & $9 q 34.2$ & DNA binding, nucleobase, nucleoside, nucleotide and nucleic acid metabolic process \\
\hline H1F0 & $22 q 13.1$ & $\begin{array}{l}\text { Nucleobase, nucleoside, nucleotide and nucleic acid metabolic process, organelle organization, establishment or maintenance } \\
\text { of chromatin architecture }\end{array}$ \\
\hline HLA-DOA & $6 \mathrm{p} 21.32$ & Antigen processing and presentation of peptide or polysaccharide antigen via MHC class II, cellular defense response \\
\hline HPD & $12 q 24.31$ & Cellular amino acid and derivative metabolic process \\
\hline HRB2 & $12 q 21.2$ & RNA binding, nucleobase, nucleoside, nucleotide and nucleic acid metabolic process \\
\hline KDELR2 & $7 p 22.1$ & Intracellular protein transport, exocytosis \\
\hline KLK8 & $19 q 13.41$ & $\begin{array}{l}\text { Spermatogenesis, immune system process, cell cycle, protein metabolic process, cell cycle, ectoderm development, nervous } \\
\text { system development, blood coagulation }\end{array}$ \\
\hline KRT16 & $17 q 21.2$ & Structural constituent of cytoskeleton, ectoderm development, cellular component morphogenesis \\
\hline LCK & $1 \mathrm{p} 35.1$ & $\begin{array}{l}\text { Female gamete generation, immune system process, carbohydrate transport, apoptosis, cell cycle, cell surface receptor linked } \\
\text { signal transduction, intracellular signaling cascade, carbohydrate metabolic process, protein metabolic process, cell motion, } \\
\text { signal transduction, cell-cell signaling, cell-cell adhesion, ectoderm development, mesoderm development, embryonic } \\
\text { development, angiogenesis, nervous system development, response to stress }\end{array}$ \\
\hline LDLR & $19 p 13.2$ & female gamete generation, cell adhesion \\
\hline LPPR4 & $1 \mathrm{p} 21.2$ & Cell surface receptor linked signal transduction, phosphate metabolic process, lipid metabolic process, signal transduction \\
\hline MAG & $19 q 13.12$ & $\begin{array}{l}\text { Receptor activity, structural constituent of myelin sheath, receptor binding, B cell mediated immunity, cell surface receptor } \\
\text { linked signal transduction, cell-cell adhesion, signal transduction, cell-cell adhesion, ectoderm development, nervous system } \\
\text { development, response to stimulus }\end{array}$ \\
\hline MAGEA3 & Xq28 & Gamete generation, induction of apoptosis, cell adhesion \\
\hline MAGEE1 & Xq13.3 & Gamete generation, induction of apoptosis, cell adhesion \\
\hline MASP1 & $3 q 27.3$ & Complement activation, protein metabolic process, response to stimulus \\
\hline $\mathrm{MLX}$ & $17 q 21.2$ & DNA binding, nucleobase, nucleoside, nucleotide and nucleic acid metabolic process, transcription factor activity \\
\hline MOGAT1 & $2 q 36.1$ & Acyl-CoA metabolic process, lipid metabolic process \\
\hline MRPL37 & $1 \mathrm{p} 32.3$ & Protein metabolic process \\
\hline MRPS12 & $19 q 13.2$ & Protein metabolic process \\
\hline NAT5 & $20 p 11.23$ & Acyltransferase activity, protein metabolic process \\
\hline NDUFA2 & $5 q 31.3$ & Oxidoreductase activity, oxidative phosphorylation, respiratory electron transport chain \\
\hline PCSK4 & $19 p 13.3$ & $\begin{array}{l}\text { Peptidase activity, cell surface receptor linked signal transduction, cell-matrix adhesion, protein metabolic process, signal } \\
\text { transduction }\end{array}$ \\
\hline
\end{tabular}


Table 3 Target genes of SIM2/ETS2 and their probable function identified by in silico analysis (Continued)

\begin{tabular}{|c|c|c|}
\hline PDE6D & $2 \mathrm{q} 37.1$ & Visual perception, sensory perception, nucleobase, nucleoside, nucleotide and nucleic acid metabolic process \\
\hline PRSS8 & $16 p 11.2$ & Peptidase activity, spermatogenesis, immune system process, protein metabolic process \\
\hline RELA & $11 \mathrm{q} 13.1$ & $\begin{array}{l}\text { B cell mediated immunity, negative regulation of apoptosis, cell cycle, intracellular signaling cascade, nucleobase, nucleoside, } \\
\text { nucleotide and nucleic acid metabolic process, signal transduction, cellular defense response }\end{array}$ \\
\hline RER1 & $1 \mathrm{p} 36.32$ & Intracellular protein transport \\
\hline S100A8 & $1 \mathrm{q} 21.3$ & $\begin{array}{l}\text { Calcium ion binding, receptor binding, calmodulin binding, immune response, macrophage activation, cell cycle, intracellular } \\
\text { signaling cascade, cell motion, cell cycle, signal transduction, response to stimulus }\end{array}$ \\
\hline S100A9 & $1 \mathrm{q} 21.3$ & $\begin{array}{l}\text { Immune response, macrophage activation, cell cycle, intracellular signaling cascade, cell motion, cell cycle, signal transduction, } \\
\text { response to stimulus }\end{array}$ \\
\hline SCARB1 & $12 q 24.31$ & $\begin{array}{l}\text { Receptor activity, macrophage activation, lipid transport, apoptosis, signal transduction, cell adhesion, lipid metabolic process, } \\
\text { cellular component morphogenesis }\end{array}$ \\
\hline SERPINA1 & $14 q 32.13$ & Protein metabolic process \\
\hline SFRS1 & $17 q 22$ & $\begin{array}{l}\text { RNA splicing factor activity, transesterification mechanism, RNA binding, nucleobase, nucleoside, nucleotide and nucleic acid } \\
\text { metabolic process }\end{array}$ \\
\hline SLC25A21 & $14 q 13.3$ & $\begin{array}{l}\text { Cation transport, phosphate transport, lipid transport, nucleobase, nucleoside, nucleotide and nucleic acid transport, } \\
\text { phosphate metabolic process, nucleobase, nucleoside, nucleotide and nucleic acid metabolic process, lipid metabolic process }\end{array}$ \\
\hline SLC7A9 & $19 q 13.11$ & $\begin{array}{l}\text { Amino acid transmembrane transporter activity, transmembrane transporter activity amino acid transport, cellular amino acid } \\
\text { and derivative metabolic process }\end{array}$ \\
\hline SP1 & $12 q 13.13$ & Immune system process, nucleoside, nucleotide and nucleic acid metabolic process \\
\hline TGM2 & $20 q 11.23$ & Protein metabolic process \\
\hline $\mathrm{TH}$ & $11 \mathrm{p} 15.5$ & Oxidoreductase activity, signal transduction, cellular amino acid and derivative metabolic process \\
\hline THBS1 & $15 q 14$ & Receptor binding, enzyme regulator activity, immune system process, blood coagulation \\
\hline TRFP & $6 \mathrm{p} 21.1$ & Ubiquitin-protein ligase activity \\
\hline TRRAP & $7 q 22.1$ & $\begin{array}{l}\text { Immune system process, induction of apoptosis, cell cycle, nucleobase, nucleoside, nucleotide and nucleic acid metabolic } \\
\text { process, protein metabolic process, cell cycle, signal transduction, organelle organization, establishment or maintenance of } \\
\text { chromatin architecture, response to stress }\end{array}$ \\
\hline
\end{tabular}

information gain (IG). Power of all chi square tests was calculated by Piface program [37]. Genotype data of four fSNPs (rs461155, rs1051425 in ETS2 and rs2073601, rs2073416 in SIM2) were also included for LD, haplotype and SNP-SNP interaction analysis. For convenience, triplicate homozygous genotypes were considered as diploid homozygous genotypes in DS probands while the triplicate heterozygous genotypes were considered as the diploid heterozygous genotype for all the calculations to compare with respective reference diploid groups [32,38].

\section{Results}

In Silico analysis to predict pathways regulated by SIM2 and ETS2

Computational expression analysis by GNFSymAtlas showed that all the splice variants of SIM2 and ETS2 were over expressed in 13 tissues and under expressed in 12 tissues (Additional file 1: Table S1). Both SIM2 and ETS2 binding sites were identified in 464 genes by the 'Consensus-Finder' program from the eukaryotic promoter database. These putative target genes of SIM2 and ETS2 were sorted into four groups (Additional file 1: Table S2). Gene set I contains 71 genes, which showed over expression in all the tissues where SIM2 and ETS2 were also over expressed. Gene set II comprised of 9 genes, which showed down regulation in all the tissues where SIM2 and ETS2 were also down regulated. The $3^{\text {rd }}$ and $4^{\text {th }}$ set of genes exhibited reverse pattern of expression as compared to SIM2 and ETS2. In addition, SP1 and AP2 were identified as common TFs for both SIM2 and ETS2 target genes.

Target pathway identification by Panther and KEGG (Table 3) indicated genes involved in several pathways, including ones related to the development of ectoderm and nervous system (KLK8, LCK, MAG), sensory perception (PDE6D), ionic transport (ATP1A1, ATP1A4, FXYD5, SLC25A2), signal transduction and cell-cell signaling (C1QL1, C4BPA, C4BPB, CEACAM1, FXYD5, LCK, GNB2L1), cell adhesion (MAGEA3, MAGEE1, MAG, LDLR, EMCN, CEACAM1, C4BPB, C4BPA, C1QL1) and induction of apoptosis (LCK, MAGEA3, MAGEE1, PRSS8, SCARB1, TRRAP). Genes governing pathways connected to immunological regulation included ABCB8, ABP1, KLK8, LCK, MAG, PRSS8, RELA, S100A9, SP1, THBS1, TRRAP, and HLA-DOA. Interestingly, genes like KLK8, LCK, RELA, S100A8, S100A9, TRRAP, and GATA3 are known to have roles in malignant development.

\section{In silico identification of functional variants}

Different in silico tools identified functional genetic variants in SIM2 and ETS2. Among them, thirty five (seven 
Table 4 Minor allele frequencies in different populations as compared to the Indian control individuals

\begin{tabular}{|c|c|c|c|c|c|c|c|c|c|}
\hline SNP ID & IND MAF & CEU MAF & Chi Sq, p & HCB MAF & Chi Sq, p & JPT MAF & Chi Sq, p & YRI MAF & Chi Sq, p \\
\hline rs2269188 & 0.348 & 0.305 & $0.570,0.450$ & 0.567 & $9.74,0.002$ & 0.534 & $6.57,0.010$ & 0.092 & $19.7,0.000$ \\
\hline rs374575 & 0.066 & 0.29 & $16.4,0.000$ & 0.14 & $2.61,0.106$ & 0.041 & $0.866,0.352$ & 0.005 & $4.69,0.030$ \\
\hline rs2070529 & 0.407 & 0.168 & $14.0,0.000$ & 0.523 & $2.43,0.119$ & 0.61 & $8.000,0.005$ & 0.27 & $4.37,0.037$ \\
\hline rs2070530 & 0.417 & 0.168 & $15.0,0.000$ & 0.523 & $2.01,0.157$ & 0.61 & $8.000,0.005$ & 0.29 & $3.69,0.055$ \\
\hline rs2070531 & 0.293 & 0.398 & $2.20,0.138$ & 0.221 & $1.29,0.256$ & 0.227 & $0.936,0.333$ & 0.288 & $0.00,1.000$ \\
\hline rs6517481 & 0.293 & 0.361 & 0.814 .0 .367 & \multicolumn{2}{|c|}{$0.318(\mathrm{HCB}+\mathrm{JPT})$} & \multicolumn{2}{|c|}{$0.212,0.645$} & 0.3 & $0.240 \mathrm{E}-01,0.877$ \\
\hline rs7276961 & 0.293 & 0.347 & $0.570,0.450$ & \multicolumn{2}{|c|}{$0.466(\mathrm{HCB}+\mathrm{JPT})$} & \multicolumn{2}{|c|}{$6.88,0.009$} & 0.3 & $0.240 \mathrm{E}-01,0.877$ \\
\hline rs1051475 & 0.276 & 0.347 & $1.14,0.287$ & \multicolumn{2}{|c|}{$0.227(\mathrm{HCB}+\mathrm{JPT})$} & \multicolumn{2}{|c|}{$0.658,0.417$} & 0.2 & $1.75,0.185$ \\
\hline rs1051476 & 0.276 & 0.42 & $4.31,0.038$ & 0.239 & $0.416,0.519$ & 0.209 & $1.32,0.25$ & 0.246 & $0.231,0.631$ \\
\hline rs11254 & 0.28 & 0.432 & $4.91,0.027$ & 0.233 & $0.658,0.417$ & 0.2 & $1.75,0.185$ & 0.25 & $0.231,0.631$ \\
\hline rs711 & 0.26 & 0.425 & $6.39,0.011$ & \multicolumn{2}{|c|}{$0.2(\mathrm{HCB}+\mathrm{JPT})$} & \multicolumn{2}{|c|}{$1.02,0.313$} & 0.441 & $7.12,0.008$ \\
\hline
\end{tabular}

MAF: Minor Allele Frequency; CEU: Caucasian population; HCB: Chinese from Beijing, China; JPT: Japanese from Tokyo, Japan; YRI: Yoruba from Ibadan, Nigeria.

in SIM2 and twenty eight in ETS2) SNPs were genotyped in this study. Functional significance of the SNPs is indicated in Table 1.

\section{Allelic and genotypic frequency distribution}

Comparative analysis of MAF in different populations revealed significant difference in many SNPs (rs374575, rs2070529, rs2070530, rs1051476, rs11254 and rs711 in CEU; rs2269188 and rs7276961 in HCB; rs2269188, rs2070529 and rs2070530 in JPT; rs2269188, rs374575, rs2070529 and rs711 in YRI) (Table 4). Among seven SNPs studied in SIM2 (Table 1), only rs2269188 was polymorphic in the studied population. This SNP showed significant difference in allelic $\left(\chi^{2}=6.333, \mathrm{P}=0.012\right.$, Power $\left.=82.3 \%\right)$ and genotypic $\left(\chi^{2}=6.41, \mathrm{P}=0.041\right.$, Power $\left.=74.17 \%\right)$ frequency only in ALL compared to the control (Additional file 1: Table S3). However, the differences were not significant after correction for multiple testing.

Twenty eight SNPs in ETS2 genomic region were analyzed and ten of them were polymorphic in the studied population. Eight of the ten ETS2 SNPs (rs374575, rs2070529, rs2070530, rs2070531, rs6517481, rs7276961, rs1051475 and rs1051476) did not show any significant difference in allelic frequency in DS probands, their parent and malignant groups (Additional file 1: Table S3). rs11254 showed a marginal allelic association in DS probands $(\mathrm{P}=0.04712)$ which failed to stand Bonferroni (BF) and Benjamini-Hochberg (BH) correction for multiple testing (Table 5). rs711 showed significant increase in the ' $\mathrm{G}$ ' allele frequency in probands with DS $\left(\chi^{2}=8.51\right.$, BF P and $\mathrm{BH} \mathrm{P}=0.03$, Power $=43.47 \%$ ) as compared to controls. Although a significant increase in the ' $\mathrm{G}$ ' allele $\left(\chi^{2}=6.83\right.$, $\mathrm{P}=0.00895$, Power $=85.03 \%$, OR $=2.6$ ) was noticed in ALL patients, it was found to be marginally significant after correction for multiple testing $(\mathrm{BH} \mathrm{P}=0.06)$. On the other hand, a significant increase in the 'A' allele $\left(\chi^{2}=9.91\right.$, BF P and $\mathrm{BH} \mathrm{P}=0.01$, Power $=88.26 \%$ ) was observed in $\mathrm{BC}$ patients (Table 5).

Significant differences in genotypic frequency for rs11254 $\left(\chi^{2}=85.4, \quad \mathrm{P}=0.0001\right.$, Power $\left.=99.96 \%\right)$, rs2070530 $\left(\chi^{2}=\right.$

Table 5 SNPs exhibiting significant differences in allelic and genotypic distribution

\begin{tabular}{|c|c|c|c|c|c|c|c|c|c|}
\hline \multirow[t]{2}{*}{ Group } & \multirow[b]{2}{*}{ SNP ID (A1/A2) } & \multicolumn{5}{|c|}{ Allelic association } & \multicolumn{3}{|c|}{ Genotypic association } \\
\hline & & CP & BFP & BHP & OR $(\mathrm{Cl})$ for $\mathrm{A} 1$ & OR $(\mathrm{Cl})$ for $\mathrm{A} 2$ & CP & BFP & BHP \\
\hline Father of DS proband $(\mathrm{N}=91)$ & $\mathrm{rs} 711(\mathrm{G} / \mathrm{A})$ & 0.019 & 0.209 & 0.215 & $0.622(0.417-0.928)$ & $1.608(1.078-2.398)$ & 0.002 & 0.022 & 0.022 \\
\hline Mother of DS proband $(\mathrm{N}=118)$ & $\mathrm{rs} 711(\mathrm{G} / \mathrm{A})$ & 0.00198 & 0.02178 & 0.022 & $0.56(0.387-0.810)$ & $1.785(1.234-2.581)$ & 0.001 & 0.011 & 0.011 \\
\hline \multirow[t]{5}{*}{ DS proband $(\mathrm{N}=132)$} & rs2070530 (C/G) & 0.892 & 1 & 0.892 & $1.024(0.729-1.438)$ & $0.977(0.695-1.372)$ & 0.003 & 0.033 & 0.015 \\
\hline & $\mathrm{rs} 1051475$ (T/C) & 0.314 & 1 & 0.576 & $0.829(0.576-1.194)$ & $1.206(0.837-1.736)$ & 0.026 & 0.286 & 0.057 \\
\hline & $\mathrm{rs} 1051476(\mathrm{C} / \mathrm{G})$ & 0.231 & 1 & 0.527 & $0.801(0.557-1.152)$ & $1.249(0.868-1.796)$ & 0.014 & 0.154 & 0.039 \\
\hline & rs11254 (C/T) & 0.04712 & 0.51832 & 0.259 & $1.487(1.004-2.201)$ & $0.673(0.454-0.996)$ & 0.0001 & 0.0011 & 0.001 \\
\hline & rs711 (G/A) & 0.00352 & 0.03872 & 0.039 & $1.858(1.221-2.828)$ & $0.538(0.354-0.819)$ & 0.004 & 0.044 & 0.015 \\
\hline \multirow[t]{2}{*}{ ALL $(\mathrm{N}=38)$} & rs2269188 (G/C) & 0.01185 & 0.13035 & 0.065 & 2.17 (1.175-4.008) & $0.461(0.250-0.851)$ & 0.041 & 0.451 & 0.226 \\
\hline & rs711 (G/A) & 0.00895 & 0.09845 & 0.065 & $2.617(1.245-5.501)$ & $0.382(0.182-0.803)$ & 0.02 & 0.22 & 0.220 \\
\hline $\mathrm{BC}(\mathrm{N}=49)$ & rs711 (G/A) & 0.00165 & 0.01815 & 0.018 & $0.469(0.291-0.755)$ & $2.133(1.324-3.437)$ & 0.002 & 0.022 & 0.022 \\
\hline$O C(N=54)$ & rs711 (G/A) & 0.04993 & 0.54923 & 0.549 & $1.758(0.995-3.105)$ & $0.569(0.322-1.005)$ & 0.091 & 1 & 0.686 \\
\hline
\end{tabular}

$B F P=$ Bonferroni $\mathrm{P}$ value, $B H P=$ Benjamini-Hochberg $\mathrm{P}$ value, $C P=$ Crude $\mathrm{P}$ value, $\mathrm{Cl}=95 \%$ Confidence Interval, $O R=$ Odds Ratio. 
Table 6 Pair wise LD pattern of studied SNPs

\begin{tabular}{|c|c|c|c|c|c|c|c|c|c|}
\hline $\mathrm{D}^{\prime}$ & rs6517481 & rs7276961 & rs1051475 & rs1051476 & rs2070529 & rs2070530 & rs2070531 & rs461155 & rs11254 \\
\hline$\overline{r^{2}}$ & & & & & & & & & \\
\hline
\end{tabular}

\begin{tabular}{|c|c|c|c|c|c|c|c|c|c|}
\hline \multicolumn{10}{|c|}{ Control } \\
\hline rs6517481 & - & 1 & 0.926 & 0.926 & 0.909 & 0.930 & 0.949 & 0.735 & 0.719 \\
\hline rs7276961 & 1 & - & 0.926 & 0.926 & 0.909 & 0.930 & 0.949 & 0.735 & 0.719 \\
\hline rs 1051475 & 0.774 & 0.774 & - & 1 & 0.899 & 0.923 & 0.944 & 0.765 & 0.722 \\
\hline rs1051476 & 0.774 & 0.774 & 1 & - & 0.899 & 0.923 & 0.944 & 0.765 & 0.722 \\
\hline rs2070529 & 0.499 & 0.499 & 0.441 & 0.441 & - & 1 & 0.955 & 0.836 & 0.660 \\
\hline rs2070530 & 0.501 & 0.501 & 0.445 & 0.445 & 0.958 & - & 0.977 & 0.849 & 0.680 \\
\hline rs2070531 & 0.902 & 0.902 & 0.805 & 0.805 & 0.551 & 0.553 & - & 0.757 & 0.718 \\
\hline rs461155 & 0.366 & 0.366 & 0.362 & 0.362 & 0.622 & 0.615 & 0.389 & - & 0.612 \\
\hline rs11254 & 0.491 & 0.491 & 0.503 & 0.503 & 0.250 & 0.255 & 0.490 & 0.240 & - \\
\hline \multicolumn{10}{|c|}{ Father of DS proband } \\
\hline rs6517481 & - & 1 & 0.856 & 0.853 & 0.871 & 0.954 & 0.905 & 0.907 & 0.845 \\
\hline rs7276961 & 1 & - & 0.856 & 0.853 & 0.871 & 0.954 & 0.905 & 0.907 & 0.845 \\
\hline rs1051475 & 0.579 & 0.579 & - & 1 & 0.710 & 0.797 & 0.888 & 0.799 & 0.897 \\
\hline rs1051476 & 0.560 & 0.560 & 0.974 & - & 0.717 & 0.802 & 0.886 & 0.805 & 0.931 \\
\hline rs2070529 & 0.423 & 0.423 & 0.369 & 0.387 & - & 0.974 & 0.956 & 0.807 & 0.876 \\
\hline rs2070530 & 0.450 & 0.450 & 0.399 & 0.415 & 0.841 & - & 1 & 0.848 & 1 \\
\hline rs2070531 & 0.796 & 0.796 & 0.606 & 0.586 & 0.494 & 0.479 & - & 0.903 & 0.905 \\
\hline rs461155 & 0.417 & 0.417 & 0.398 & 0.414 & 0.579 & 0.703 & 0.400 & - & 0.861 \\
\hline rs11254 & 0.714 & 0.714 & 0.643 & 0.674 & 0.442 & 0.494 & 0.795 & 0.376 & - \\
\hline \multicolumn{10}{|c|}{ Mother of DS proband } \\
\hline rs6517481 & - & 1 & 0.828 & 0.830 & 0.919 & 0.918 & 0.979 & 0.769 & 0.916 \\
\hline rs7276961 & 1 & - & 0.828 & 0.830 & 0.919 & 0.918 & 0.979 & 0.769 & 0.916 \\
\hline rs 1051475 & 0.549 & 0.549 & - & 1 & 0.658 & 0.671 & 0.849 & 0.548 & 0.926 \\
\hline rs1051476 & 0.563 & 0.563 & 0.981 & - & 0.679 & 0.693 & 0.849 & 0.571 & 0.927 \\
\hline rs2070529 & 0.538 & 0.568 & 0.346 & 0.362 & - & 1 & 1 & 0.745 & 0.918 \\
\hline rs2070530 & 0.528 & 0.528 & 0.346 & 0.363 & 0.982 & - & 1 & 0.750 & 0.916 \\
\hline rs2070531 & 0.920 & 0.920 & 0.552 & 0.565 & 0.612 & 0.601 & - & 0.759 & 0.937 \\
\hline rs461155 & 0.370 & 0.370 & 0.228 & 0.243 & 0.535 & 0.563 & 0.346 & - & 0.728 \\
\hline rs11254 & 0.823 & 0.823 & 0.663 & 0.677 & 0.527 & 0.515 & 0.861 & 0.316 & - \\
\hline \multicolumn{10}{|c|}{ DS proband } \\
\hline rs6517481 & - & 1 & 0.865 & 0.862 & 0.875 & 0.975 & 0.939 & 0.761 & 0.255 \\
\hline rs7276961 & 1 & - & 0.865 & 0.862 & 0.875 & 0.975 & 0.939 & 0.761 & 0.255 \\
\hline rs 1051475 & 0.710 & 0.710 & - & 1 & 0.830 & 0.926 & 0.903 & 0.774 & 0.274 \\
\hline rs1051476 & 0.680 & 0.680 & 0.966 & - & 0.807 & 0.903 & 0.885 & 0.756 & 0.266 \\
\hline rs2070529 & 0.501 & 0.501 & 0.476 & 0.458 & - & 0.949 & 0.825 & 0.812 & 0.256 \\
\hline rs2070530 & 0.593 & 0.593 & 0.565 & 0.546 & 0.859 & - & 0.911 & 0.823 & 0.273 \\
\hline rs2070531 & 0.754 & 0.754 & 0.735 & 0.718 & 0.523 & 0.608 & - & 0.699 & 0.269 \\
\hline rs461155 & 0.375 & 0.375 & 0.410 & 0.404 & 0.659 & 0.645 & 0.376 & - & 0.167 \\
\hline rs11254 & 0.039 & 0.039 & 0.043 & 0.039 & 0.026 & 0.028 & 0.037 & 0.011 & - \\
\hline \multicolumn{10}{|c|}{ ALL } \\
\hline rs6517481 & - & 1 & 1 & 1 & 1 & 1 & 1 & 0.761 & 1 \\
\hline rs7276961 & 1 & - & 1 & 1 & 1 & 1 & 1 & 0.761 & 1 \\
\hline rs1051475 & 0.939 & 0.939 & - & 1 & 0.926 & 1 & 1 & 0.698 & 1 \\
\hline rs 1051476 & 0.939 & 0.939 & 1 & - & 0.926 & 1 & 1 & 0.698 & 1 \\
\hline rs2070529 & 0.698 & 0.698 & 0.638 & 0.638 & - & 1 & 0.926 & 0.748 & 0.858 \\
\hline
\end{tabular}


Table 6 Pair wise LD pattern of studied SNPs (Continued)

\begin{tabular}{|c|c|c|c|c|c|c|c|c|c|}
\hline rs2070530 & 0.625 & 0.625 & 0.665 & 0.665 & 0.894 & - & 1 & 0.731 & 1 \\
\hline rs2070531 & 0.939 & 0.939 & 1 & 1 & 0.638 & 0.665 & - & 0.698 & 1 \\
\hline rs461155 & 0.428 & 0.428 & 0.383 & 0.383 & 0.529 & 0.451 & 0.383 & - & 0.636 \\
\hline rs11254 & 0.883 & 0.883 & 0.940 & 0.940 & 0.582 & 0.708 & 0.940 & 0.339 & - \\
\hline \multicolumn{10}{|c|}{$B C$} \\
\hline rs6517481 & - & 1 & 0.884 & 0.884 & 1 & 1 & 1 & 0.5 & 0.122 \\
\hline rs7276961 & 1 & - & 0.884 & 0.884 & 1 & 1 & 1 & 0.5 & 0.122 \\
\hline rs1051475 & 0.741 & 0.741 & - & 1 & 1 & 1 & 0.884 & 0.891 & 0.077 \\
\hline rs1051476 & 0.741 & 0.741 & 1 & - & 1 & 1 & 0.884 & 0.891 & 0.077 \\
\hline rs2070529 & 0.631 & 0.631 & 0.605 & 0.605 & - & 1 & 1 & 0.495 & 0.274 \\
\hline rs2070530 & 0.605 & 0.60 & 0.582 & 0.582 & 1 & - & 1 & 0.495 & 0.269 \\
\hline rs2070531 & 1 & 1 & 0.741 & 0.741 & 0.631 & 0.605 & - & 0.5 & 0.122 \\
\hline rs461155 & 0.125 & 0.125 & 0.350 & 0.350 & 0.204 & 0.204 & 0.125 & - & 0.425 \\
\hline rs11254 & 0.013 & 0.013 & 0.001 & 0.001 & 0.041 & 0.038 & 0.013 & 0.088 & - \\
\hline \multicolumn{10}{|c|}{ OC } \\
\hline rs6517481 & - & 1 & 0.948 & 0.948 & 1 & 1 & 1 & 0.334 & 0.899 \\
\hline rs7276961 & 1 & - & 0.948 & 0.948 & 1 & 1 & 1 & 0.334 & 0.899 \\
\hline rs1051475 & 0.697 & 0.697 & - & 1 & 1 & 1 & 0.950 & 0.632 & 0.907 \\
\hline rs1051476 & 0.697 & 0.697 & 1 & - & 1 & 1 & 0.950 & 0.632 & 0.907 \\
\hline rs2070529 & 0.607 & 0.607 & 0.471 & 0.471 & - & 1 & 1 & 0.391 & 0.856 \\
\hline rs2070530 & 0.630 & 0.630 & 0.488 & 0.488 & 0.963 & - & 1 & 0.306 & 0.861 \\
\hline rs2070531 & 0.960 & 0.960 & 0.728 & 0.728 & 0.582 & 0.605 & - & 0.306 & 0.902 \\
\hline rs461155 & 0.060 & 0.060 & 0.168 & 0.168 & 0.137 & 0.049 & 0.049 & - & 0.656 \\
\hline rs11254 & 0.656 & 0.656 & 0.787 & 0.787 & 0.360 & 0.378 & 0.687 & 0.189 & - \\
\hline
\end{tabular}

11.5, $\mathrm{P}=0.003$, Power $=44.76 \%)$, rs1051475 $\left(\chi^{2}=7.33\right.$, $\mathrm{P}=0.026, \quad$ Power $=30.01 \%), \quad \operatorname{rs} 1051476 \quad\left(\chi^{2}=8.57\right.$, $\mathrm{P}=0.014$, Power $=34.5 \%)$ and $\operatorname{rs} 711\left(\chi^{2}=11.2, \mathrm{P}=0.004\right.$, Power $=43.74 \%$ ) were observed in DS probands. The heterozygous genotype frequency of rs11254 was found to be 0.000 in DS probands. rs711 also showed significant difference in genotype distribution in parents of probands with DS (Father: $\chi^{2}=12.8, \mathrm{P}=0.002$, Power $=$ 65.76\%; Mother: $\chi^{2}=14.6, \mathrm{P}=0.001$, Power $\left.=59.81 \%\right)$ as well as in ALL $\left(\chi^{2}=7.78, \mathrm{P}=0.02\right.$, Power $\left.=82.49 \%\right)$ and $\mathrm{BC}\left(\chi^{2}=12.1, \mathrm{P}=0.002\right.$, Power $\left.=89.24 \%\right)$. 'AA' genotype was absent in the ALL and OC groups. Except for rs1051475 in probands with DS and rs711 in ALL patients, the remaining SNPs retained the significance level after BF and BH correction (Table 5).

\section{LD and haplotype analysis}

SNP pairs that showed higher LD (high $D^{\prime}$ or $r^{2}$ value) in at least one combination or different LD patterns in control and case groups during pair wise analysis by Haploview 4.1 were sorted out. In control individuals and parents of probands with DS, all the studied SNPs exhibited strong LD (Table 6). In particular, rs6517481rs7276961, rs1051475-rs1051476, rs2070529-rs2070530, rs2070531-rs6517481, rs2070531-rs7276961 pairs exhibited strong LD in other studied groups. Some paired combinations showed different LD pattern in different disease groups. For instance, rs11254 showed weak LD with all the sites in DS and BC groups, while rs461155 showed weak LD in OC. Statistically significant differences in frequency of several haplotypes were noticed between test and control groups (Figure 2). Notably the 'A-C-CC-T-C-C-A-A-T-C-C-C-G-G' haplotype showed significant frequency difference in $\mathrm{BC}$, DS proband and their parents when analyzed by Unphased. However, comparison by simple Chi-square test followed by analysis of the power of association by Piface (Additional file 1: Table S4) showed statistically significant difference only for the DS probands and BC groups (p value 0.054 and 0.013 respectively).

\section{Analysis of gene-gene interaction}

Gene-gene interaction analysis by MDR 2.0 beta 8.1 indicated that different combinations of SNPs were interacting with each other in different ways within these groups. No highly synergistic interaction was observed in DS probands, while individual effect of different SNPs were found to be high in DS [rs2073601 (1.92\%), rs461155 (2.64\%), 


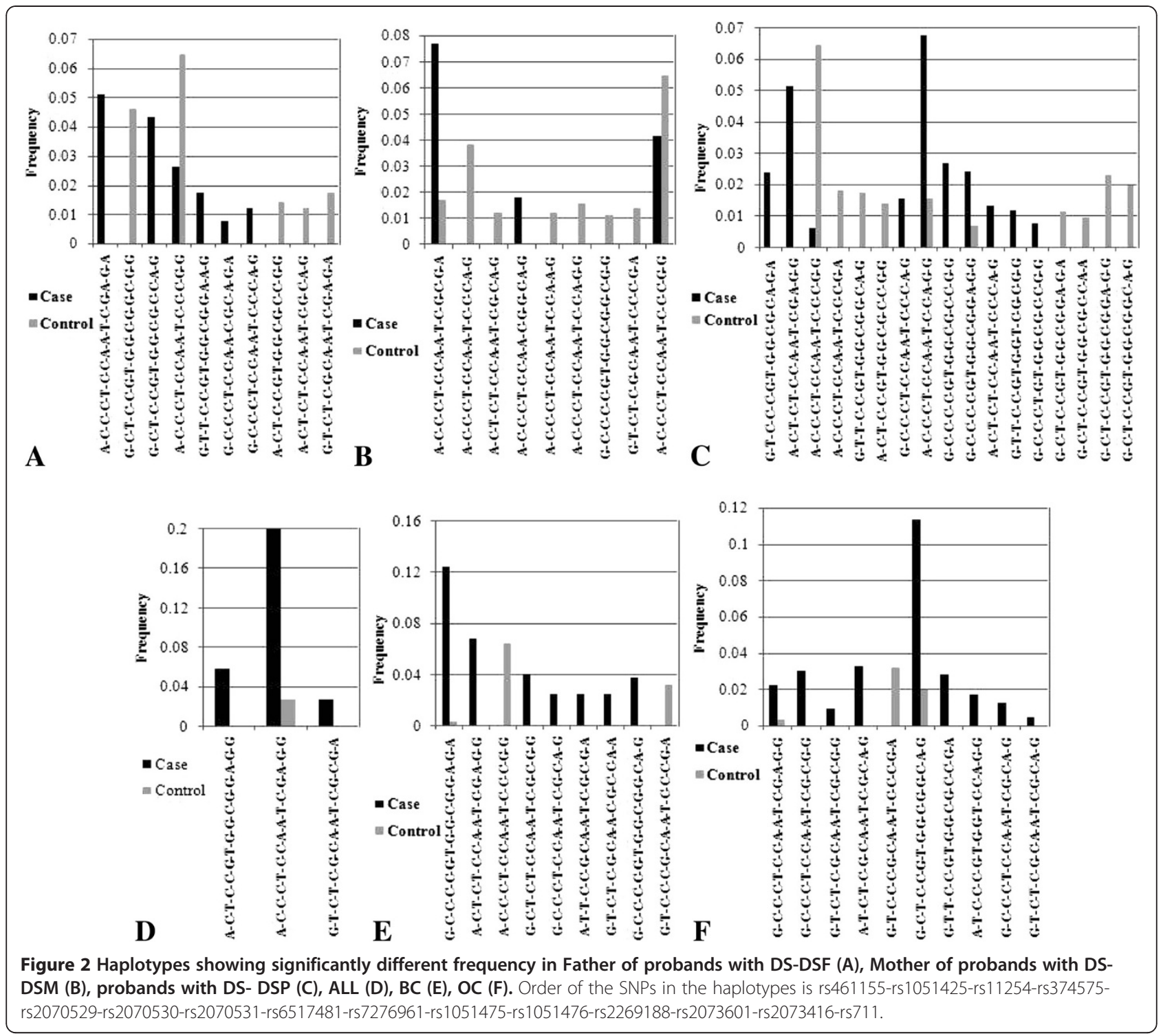

rs1051425 (1.21\%), rs2070529 (1.47\%), rs2070530 (3.04\%), rs2070531 (1.09\%), rs6517481 (1.66\%), rs7276961 (1.66\%), rs1051475 (2.24\%), rs1051476 (2.56\%), rs11254 (29.78\%) and rs711 (4.26\%)] (Table 7).

While there was no high individual effect of rs2070529, rs2070530, rs2070531, rs6517481 and rs7276961, high synergistic interaction of these SNPs with rs11254 was noticed in mother of probands with DS (Table 7). In father of probands with DS, rs2070531, rs6517481 and rs7276961 also made a cluster together with rs2073416. High individual effect of rs2073601 (1.77\%), rs1051475 (2.09\%), rs1051476 (2.29\%) and rs711 (3.08\%) was observed in father of probands with DS (Table 7), while rs2073601 (1.38\%), rs2073416 (1.96\%), rs2269188 (17.17\%), $\quad$ rs461155
(1.06\%), rs1051425 (1.11\%), $\quad$ rs374575 (1.10\%), rs1051475 (1.47\%), rs1051476 (1.34\%) and rs711 $(4.03 \%)$ showed high individual effect in mothers (Table 7).

In the malignant groups, rs2070530, rs2070531, rs6517481, rs7276961, rs1051475, rs1051476 and rs11254 showed synergistic interaction (IG values are mentioned in Table 8). Many of the studied fSNPs also showed significant individual effect in ALL [rs2073601 (3.69\%), rs2073416 (2.95\%), rs2269188 (2.56\%), rs374575 (1.03\%) and rs711 (3.55\%)], BC [rs2073601 (3.50\%), rs2073416 (2.17\%), rs461155 (15.15\%), rs374575 (4.04\%), rs2070529 (3.79\%), rs2070530 (3.03\%), rs2070531 (4.01\%), rs6517481 (3.88\%), rs7276961 (3.88\%), rs1051475 (1.00\%), rs1051476 (1.00\%), rs11254 (1.12\%) and rs711 (4.56\%)] and OC [rs2073601 
Table 7 Individual and interactive effects of SIM2 and ETS2 SNPs in families with DS probands (analyzed by MDR 2.0 beta 8.1)

\begin{tabular}{|c|c|c|c|c|c|c|c|c|c|c|c|c|c|c|c|}
\hline \multirow{2}{*}{$\begin{array}{l}\text { SNP ID (individual } \\
\text { effect) }\end{array}$} & \multicolumn{15}{|c|}{ Interactive effects } \\
\hline & 1 & 2 & 3 & 4 & 5 & 6 & 7 & 8 & 9 & 10 & 11 & 12 & 13 & 14 & 15 \\
\hline \multicolumn{16}{|c|}{ Father } \\
\hline rs2073601(1.77) & - & -0.06 & -1.00 & -0.72 & -1.49 & -1.07 & -1.22 & -0.82 & -1.24 & -0.99 & -0.99 & -0.09 & -0.08 & -1.12 & -1.79 \\
\hline rs2073416 (0.94) & & - & -0.65 & -0.05 & -0.73 & -1.12 & 0.92 & -0.37 & 0.83 & -0.46 & -0.46 & 1.41 & 1.64 & 0.72 & -0.51 \\
\hline rs2269188 (0.22) & & & - & 1.10 & -0.54 & -0.38 & 0.55 & 1.32 & 0.50 & 0.69 & 0.69 & -0.20 & -0.07 & 0.38 & 0.12 \\
\hline rs461155 (0.70) & & & & - & -0.40 & 0.39 & -0.08 & -0.11 & 0.55 & -0.19 & -0.19 & -0.62 & -0.54 & -0.40 & -0.12 \\
\hline rs1051425 (0.38) & & & & & - & -0.58 & 0.69 & -0.18 & -0.09 & 0.55 & 0.55 & 0.09 & 0.19 & -0.19 & -0.05 \\
\hline rs374575 (0.65) & & & & & & - & -0.58 & -0.38 & -0.85 & -0.66 & -0.66 & -0.87 & -0.81 & -0.57 & -0.42 \\
\hline rs2070529 (0.17) & & & & & & & - & -0.14 & -0.43 & -0.18 & -0.18 & -0.25 & -0.18 & -0.67 & 0.06 \\
\hline rs2070530 (0.44) & & & & & & & & - & -0.41 & 0 & 0 & -0.15 & -0.06 & 1.16 & -0.21 \\
\hline rs2070531 (0.49) & & & & & & & & & - & -0.41 & -0.41 & 3.43 & 3.78 & 2.07 & 0.37 \\
\hline rs6517481 (0.34) & & & & & & & & & & - & -0.41 & 3.21 & 3.53 & 0.37 & 0.04 \\
\hline rs7276961 (0.34) & & & & & & & & & & & - & 3.21 & 3.53 & 0.37 & 0.04 \\
\hline rs1051475 (2.09) & & & & & & & & & & & & - & -2.67 & -0.69 & 0.24 \\
\hline rs1051476 (2.29) & & & & & & & & & & & & & - & -0.66 & 0.46 \\
\hline rs11254 (0.09) & & & & & & & & & & & & & & - & -0.11 \\
\hline rs711 (3.08) & & & & & & & & & & & & & & & - \\
\hline
\end{tabular}

\begin{tabular}{|c|c|c|c|c|c|c|c|c|c|c|c|c|c|c|c|}
\hline \multicolumn{16}{|c|}{ Mother } \\
\hline rs2073601 (1.38) & - & -1.49 & -2.99 & -0.16 & -1.27 & -1.38 & -0.75 & -0.62 & -0.14 & -0.09 & -0.09 & -0.67 & -0.75 & -1.31 & -1.40 \\
\hline rs2073416 (1.96) & & - & -4.27 & -0.25 & -1.45 & -1.34 & -0.17 & -0.17 & -0.45 & -0.46 & -0.46 & -0.84 & 0.03 & -0.05 & -1.64 \\
\hline rs2269188 (17.17) & & & - & -3.97 & -3.97 & -4.13 & -3.25 & -2.90 & -3.71 & -4.19 & -4.19 & -5.05 & -4.92 & -3.47 & -4.23 \\
\hline rs461155 (1.06) & & & & - & -0.36 & 0.19 & 0.02 & 0.22 & 0.44 & 0.49 & 0.49 & -0.46 & -0.53 & -0.21 & -0.84 \\
\hline rs1051425 (1.11) & & & & & - & -0.95 & 0.19 & -0.25 & -1.03 & -0.84 & -0.84 & -0.23 & -0.31 & -0.84 & -0.59 \\
\hline rs374575 (1.10) & & & & & & - & -0.75 & -0.41 & -0.56 & -0.66 & -0.66 & -0.97 & -1.03 & -0.69 & -0.02 \\
\hline rs2070529 (0.63) & & & & & & & - & -0.54 & 0.14 & 0.19 & 0.19 & -0.09 & -0.16 & 2.27 & 0.66 \\
\hline rs2070530 (0.73) & & & & & & & & - & 0.16 & 0.3 & 0.3 & 0.19 & 0.07 & 2.43 & 0.92 \\
\hline rs2070531(0.36) & & & & & & & & & - & -0.47 & -0.47 & 2.8 & 2.6 & 5.71 & 0.52 \\
\hline rs6517481 (0.30) & & & & & & & & & & - & -0.51 & 2.07 & 1.9 & 2.97 & 0.73 \\
\hline rs7276961 (0.30) & & & & & & & & & & & - & 2.07 & 1.9 & 2.97 & 0.73 \\
\hline rs1051475 (1.47) & & & & & & & & & & & & - & -1.71 & -0.72 & -1.17 \\
\hline rs1051476 (1.34) & & & & & & & & & & & & & - & -0.75 & -1.04 \\
\hline rs11254 (0.66) & & & & & & & & & & & & & & - & -0.67 \\
\hline
\end{tabular}

\begin{tabular}{|c|c|c|c|c|c|c|c|c|c|c|c|c|c|c|c|}
\hline \multicolumn{16}{|c|}{ Probands with DS } \\
\hline rs2073601 (1.92) & - & -0.37 & 0.09 & -0.34 & -0.67 & -0.26 & -0.81 & -0.57 & -1.28 & -1.14 & -1.14 & -1.83 & -1.74 & -3.83 & -2.56 \\
\hline rs2073416 (0.26) & & - & 1.10 & -0.22 & -0.46 & 0.12 & -0.17 & -0.41 & -0.39 & -1.27 & -1.27 & -0.49 & -0.43 & -2.17 & -1.72 \\
\hline rs2269188 (0.87) & & & - & 1.35 & -0.92 & -0.03 & 0.56 & -0.34 & 0.94 & -0.13 & -0.13 & -0.39 & -0.27 & -2.78 & -2.46 \\
\hline rs461155 (2.64) & & & & - & -1.08 & 0.40 & -0.99 & -2.07 & -0.58 & -1.27 & -1.27 & -1.98 & -2.08 & -11.9 & -3.42 \\
\hline rs1051425 (1.21) & & & & & - & -0.24 & -0.84 & -1.36 & -0.80 & -1.69 & -1.69 & -0.74 & -0.62 & -2.60 & -0.10 \\
\hline rs374575 (0.03) & & & & & & - & -0.23 & -0.37 & 0 & -0.76 & -0.76 & -0.48 & -0.46 & -1.94 & -0.47 \\
\hline rs2070529 (1.47) & & & & & & & - & -1.61 & -0.4 & -0.96 & -0.96 & -0.99 & -0.88 & -8.74 & -1.09 \\
\hline rs2070530 (3.04) & & & & & & & & - & -0.77 & -1.38 & -1.38 & -1.7 & -1.76 & -13.5 & -1.98 \\
\hline rs2070531 (1.09) & & & & & & & & & - & -1.03 & -1.03 & -0.8 & -0.71 & -8.71 & -1.31 \\
\hline rs6517481 (1.66) & & & & & & & & & & - & -2.73 & -1.79 & -1.71 & -5.3 & -2.77 \\
\hline rs7276961 (1.66) & & & & & & & & & & & - & -1.79 & -1.71 & -5.3 & -2.77 \\
\hline
\end{tabular}


Table 7 Individual and interactive effects of SIM2 and ETS2 SNPs in families with DS probands (analyzed by MDR 2.0 beta 8.1) (Continued)

\begin{tabular}{|c|c|c|c|}
\hline rs1051475 (2.24) & -2.68 & -7.08 & -2.15 \\
\hline rs1051476 (2.56) & - & -6.21 & -2.47 \\
\hline rs11254 (29.78) & & - & -3.58 \\
\hline rs711(4.26) & & & - \\
\hline
\end{tabular}

(2.04\%), rs461155 (13.14\%), rs374575 (1.89\%), rs2070529

(1.16\%), rs2070531 (1.47\%), rs6517481 (1.33\%), rs7276961

(1.33\%) and rs711 (2.26\%)] (Table 8).

\section{Discussion}

The present study was aimed at identifying possible involvement of SIM2 and ETS2, two TFs known to have gene overdosage in probands with DS exhibiting trisomy of HSA21. To identify SIM2 and ETS2 targets, we focused on 464 genes containing binding site for both these factors in their regulatory regions $(-5000 \mathrm{bp}$ to $+1000 \mathrm{bp}$ ). Following categorization based on expression pattern by GNF SymAtlas, 91 genes were identified as up- or down regulated by these TFs (Additional file 1: Table S2). Genes like ABP1, HRB2, S100A8, THBS1, CYB561, GATA1, GATA3, SP1 and AP2 indicated potential activation by SIM2 and ETS2 (gene set I and II), while genes such as GCNT2, MASP1, LOC338328, PCSK4, ICAM1, LPPR4, SLC25A21, H1F0 and ATP1A1 indicated potential repression by these TFs (gene set III and IV). Many of the genes with binding sites for SIM2 and ETS2, viz. KLK8, LCK, TRRAP, GATA3, etc. were earlier reported to have role in neurological as well as malignancy related pathways $[28,39,40]$. Analysis in the present study by Panther also revealed that genes such as KLK8, KRT16, and LCK carrying binding sites for SIM2 and ETS2, are involved in the development and function of the neurological system. Hence over expression of SIM2 and ETS2 might alter expression of the downstream target genes leading to different DS phenotypes.

Previous analysis of DS revealed ambiguous observations on expressions of genes in HSA21 and other autosomes. For instance, a dosage dependent increase in transcription across different tissue/cell types was noticed in DS [41]. Analysis of lymphoblastoid cell lines generated from unrelated individuals revealed over expression of several HSA21 genes even in normal healthy volunteers [42]. In contrast, gene expression profile analysis of hearts of human fetuses with trisomy of HSA21 showed significant downregulation of 278 genes and upregulation of 195 genes as compared to controls [43]. On the other hand, serial analysis of gene expression in lymphocytes from children with DS revealed modest deregulation of autosomal genes [44]. Whole genome microarray in adult DS brains showed upregulation of $27 \%$ of genes on HSA21 as compared to $4.4 \%$ of genes on other autosomes [45]. Contrary to that, microarray analysis of cultured amniocytes and chorionic villus cells from fetuses with trisomy 13, 18, or 21 revealed lack of over expression of most of the HSA21 genes with only modest changes for genes on all other chromosomes [46]. It is possible that the differences in gene expression in HSA21 and other autosomes are due to the tissue of origin [47].

Differential expression of SIM2 and ETS2 target genes was also reported in different malignancies. For instance, the TRRAP gene, involved in transcriptional regulation and DNA repair, was found to be high in bone metastases from prostate cancer, intermediate in $\mathrm{BC}$, and low in lung and kidney cancers [39]. KLK8 was upregulated in colorectal cancer and ovarian cancer while underexpressed in esophageal and cervical cancer $[40,48]$. Differential gene expression profiling of approximately 8000 genes in sixty different cancer cell lines revealed difference in gene expression pattern to be correlated with the tissue of origin and the physiological properties (e.g., doubling time, drug metabolism, and interferon response) of cell lines [49]. Difference in expression between specific cancer cell line and their nonmalignant counterparts was also noticed [49]. It is intriguing to note that genes like MAGEA3 and ATP1A1, which indicated potential over expression in our study, are also over expressed in leukemia/lymphoma [48,50-52]. However, THBS1, which also indicated potential upregulation in the present study, was down regulated in leukemia and upregulated in lymphoma $[48,53]$. Thus, it remains unclear whether differential expression is also taking place for genes identified by our present in silico analysis. Further validation, involving expression analysis in various tumor tissues of individuals with DS, will be necessary.

Our next goal was to identify fSNPs in these two TFs. A number of SNPs with deleterious effects were identified in both the genes by our in silico approach. Analysis of allelic frequencies showed significant difference in MAF for the Indian control population as compared to other Asian, i.e. Japanese and Chinese, as well as Caucasian populations. Frequency distribution analysis revealed that the rs2269188 ' $G$ ' allele was significantly high in ALL subjects, which failed to stand test for 
Table 8 Individual and interactive effects of SIM2 and ETS2 SNPs in different malignant groups (analyzed by MDR 2.0 beta 8.1)

\begin{tabular}{|c|c|c|c|c|c|c|c|c|c|c|c|c|c|c|c|}
\hline \multirow[b]{2}{*}{ SNP ID (individual effect) } & \multicolumn{15}{|c|}{ Interactive effects } \\
\hline & 1 & 2 & 3 & 4 & 5 & 6 & 7 & 8 & 9 & 10 & 11 & 12 & 13 & 14 & 15 \\
\hline \multicolumn{16}{|c|}{ ALL } \\
\hline rs2073601 (3.69) & - & -4.91 & -3.23 & -1.16 & -0.45 & -3.42 & -1.34 & -1.41 & -2.55 & -2.33 & -2.33 & -2.45 & -2.45 & -2.13 & -4.27 \\
\hline rs2073416 (2.95) & & - & -1.55 & -1.00 & -1.97 & -2.21 & -1.99 & -2.25 & -2.44 & -1.98 & -1.98 & -2.12 & -2.12 & -1.58 & -3.53 \\
\hline rs2269188 (2.56) & & & - & 0.74 & -0.29 & -0.01 & 0.79 & 0.16 & -0.47 & -0.25 & -0.25 & -0.22 & -0.22 & -0.31 & -2.15 \\
\hline rs461155 (0.07) & & & & - & 0.21 & -0.53 & -0.37 & 0.30 & -0.63 & -0.39 & -0.39 & -0.21 & -0.21 & -0.29 & -0.65 \\
\hline rs1051425 (0.65) & & & & & - & -0.31 & -0.13 & -0.52 & -0.06 & 0.18 & 0.18 & 0.07 & 0.07 & -0.26 & -0.74 \\
\hline rs374575 (1.03) & & & & & & - & -1.08 & -1.18 & -1.15 & -1.14 & -1.14 & -0.89 & -0.89 & -1.06 & -0.69 \\
\hline rs2070529 (0.73) & & & & & & & - & -1.04 & -0.91 & -0.16 & -0.16 & 0.48 & 0.48 & 0.91 & -1.15 \\
\hline rs2070530 (0.64) & & & & & & & & - & -1.10 & -0.90 & -0.90 & 0.15 & 0.15 & 1.87 & -1.06 \\
\hline rs2070531 (0.95) & & & & & & & & & - & -1.13 & -1.13 & 1.44 & 1.44 & 2.53 & -1.23 \\
\hline rs6517481 (0.58) & & & & & & & & & & - & -1.09 & 2.21 & 2.21 & 2.90 & -1.00 \\
\hline rs7276961 (0.58) & & & & & & & & & & & - & 2.21 & 2.21 & 2.90 & -1.00 \\
\hline rs1051475 (0.39) & & & & & & & & & & & & - & -0.57 & 2.52 & -0.97 \\
\hline rs1051476 (0.39) & & & & & & & & & & & & & - & 2.52 & -0.97 \\
\hline rs11254 (0.63) & & & & & & & & & & & & & & - & -0.97 \\
\hline 711 (3.55) & & & & & & & & & & & & & & & - \\
\hline
\end{tabular}

\begin{tabular}{|c|c|c|c|c|c|c|c|c|c|c|c|c|c|c|c|}
\hline \multicolumn{16}{|c|}{ BC } \\
\hline rs2073601 (3.50) & - & -4.62 & -3.32 & -3.69 & -3.32 & -3.33 & -3.89 & -3.12 & -3.03 & -2.66 & -2.66 & -1.97 & -1.97 & -0.76 & -3.03 \\
\hline rs2073416 (2.17) & & - & -2.10 & -2.36 & -1.86 & -1.78 & -4.05 & -3.14 & 2.60 & 2.47 & 2.47 & 1.90 & 1.90 & -0.80 & -1.56 \\
\hline rs2269188 (0.92) & & & - & -2.70 & 1.66 & 0.07 & -1.09 & -0.14 & -2.90 & -2.77 & -2.77 & -0.96 & -0.96 & -1.45 & 0.02 \\
\hline rs461155 (15.15) & & & & - & -0.77 & -3.55 & -3.99 & -3.22 & -4.20 & -4.07 & -4.07 & -1.19 & -1.19 & -1.31 & -7.00 \\
\hline rs1051425 (0.58) & & & & & - & -2.71 & -1.57 & -0.97 & -0.97 & -1.50 & -1.50 & 0.37 & 0.37 & -0.66 & -1.16 \\
\hline rs374575 (4.04) & & & & & & - & -5.88 & -5.44 & -3.83 & -4.28 & -4.28 & -2.39 & -2.39 & -2.19 & -3.01 \\
\hline rs2070529 (3.79) & & & & & & & - & -5.97 & -3.87 & -5.35 & -5.35 & -1.63 & -1.63 & -0.79 & -2.14 \\
\hline rs2070530 (3.03) & & & & & & & & - & -4.90 & -5.15 & -5.15 & -1.54 & -1.54 & 0.33 & -1.63 \\
\hline rs2070531 (4.01) & & & & & & & & & - & -4.91 & -4.91 & 2.19 & 2.19 & 3.81 & -2.37 \\
\hline rs6517481 (3.88) & & & & & & & & & & - & -5.09 & 0.86 & 0.86 & 3.94 & -1.95 \\
\hline rs7276961 (3.88) & & & & & & & & & & & - & 0.86 & 0.86 & 3.94 & -1.95 \\
\hline rs1051475 (1.00) & & & & & & & & & & & & - & -1.23 & 7.32 & 0.19 \\
\hline rs1051476 (1.00) & & & & & & & & & & & & & - & 7.32 & 0.19 \\
\hline rs11254 (1.12) & & & & & & & & & & & & & & - & -1.36 \\
\hline
\end{tabular}

\begin{tabular}{|c|c|c|c|c|c|c|c|c|c|c|c|c|c|c|c|}
\hline \multicolumn{16}{|c|}{ OC } \\
\hline rs2073601 (2.04) & - & -0.91 & -1.51 & -2.04 & -0.62 & -1.12 & -0.79 & -0.79 & -1.14 & -1.27 & -1.27 & -1.46 & -1.46 & -1.38 & -2.44 \\
\hline rs2073416 (0.04) & & - & 0.21 & -0.41 & -0.25 & -1.23 & 0.24 & 1.00 & -0.58 & -0.37 & -0.37 & 1.27 & 1.27 & 0.42 & -1.05 \\
\hline rs2269188 (0.81) & & & - & -1.17 & 0.45 & -0.52 & -0.49 & -0.35 & -0.97 & -0.94 & -0.94 & -0.17 & -0.17 & -0.26 & -1.31 \\
\hline rs461155 (13.14) & & & & - & -0.70 & -1.58 & -0.64 & -0.38 & -1.83 & -1.69 & -1.69 & 0.38 & 0.38 & -0.47 & -1.26 \\
\hline rs1051425 (0.34) & & & & & - & -1.38 & -0.77 & -0.76 & -1.37 & -1.07 & -1.07 & -0.05 & -0.05 & -0.20 & -1.23 \\
\hline rs374575 (1.89) & & & & & & - & -2.33 & -2.23 & -1.45 & -1.50 & -1.50 & -1.56 & -1.56 & -0.97 & -2.66 \\
\hline rs2070529 (1.16) & & & & & & & - & -1.34 & -1.78 & -1.47 & -1.47 & -1.05 & -1.05 & -0.35 & -1.52 \\
\hline rs2070530 (0.91) & & & & & & & & - & -1.68 & -1.31 & -1.31 & -1.92 & -1.92 & -0.44 & -1.63 \\
\hline rs2070531 (1.47) & & & & & & & & & - & -2.48 & -2.48 & 1.90 & 1.90 & 0.67 & -2.13 \\
\hline rs6517481 (1.33) & & & & & & & & & & - & -2.40 & -1.46 & -1.46 & 0.03 & -1.99 \\
\hline rs7276961 (1.33) & & & & & & & & & & & - & -1.46 & -1.46 & 0.03 & -1.99 \\
\hline rs1051475 (0.48) & & & & & & & & & & & & - & -0.69 & 1.33 & -0.73 \\
\hline
\end{tabular}


Table 8 Individual and interactive effects of SIM2 and ETS2 SNPs in different malignant groups (analyzed by MDR 2.0 beta 8.1) (Continued)

\begin{tabular}{|c|c|c|}
\hline rs1051476 (0.48) & 1.33 & -0.73 \\
\hline rs11254 (0.11) & - & -0.51 \\
\hline rs711 (2.26) & & - \\
\hline
\end{tabular}

multiple correction. Haplotypes showing significant difference in ALL, BC and OC groups harbored the rs2269188 ' $G$ ' allele. MDR analysis revealed high individual effect of this SNP in ALL (2.56\%) and in mother of DS probands (17.17\%) but not in any other groups. ' $G$ ' allele is responsible for AhR binding to SIM2. AhR binding with ARNT is an important step for carcinogen metabolism, which is inhibited by SIM2 [6-10]. We speculate that increased frequency of the rs2269188 ' $G$ ' allele may result in inappropriate metabolism of carcinogenic compounds, thus contributing to the development of leukemia.

rs711 is a site for SR protein mediated splicing regulation and may generate splice variants. In the Korean population, rs711 was reported to be associated with increased risk for acute myeloid leukemia [54]. In the present study, difference in allelic frequency for this site showed a trend to be significant in ALL even after correction for multiple testing, while DS probands, parents of DS probands and $\mathrm{BC}$ showed significant differences. MDR analysis supported evidence of individual effect of this SNP in all the studied groups.

rs11254, rs2070530 and rs1051476 showed significant difference in genotype distribution in DS probands $(\mathrm{BH}$ $\mathrm{P}=0.001,0.01$ and 0.03 respectively). Though there was individual effect of these SNPs $(29.78 \%, 3.04 \%$, and $2.56 \%$ respectively), no significant synergistic effect was observed. rs11254 showed a very high individual effect (29.78\%) in DS probands which could be due to $100 \%$ reduction in heterozygosity. On the other hand in malignant groups, rs11254 showed interactive effect in synergistic mode with other SNPs (rs2070530, rs2070531, rs6517481, rs7276961, rs1051475 and rs1051476). Therefore, this SNP may act differently in DS and other malignant groups.

While comparing differences in haplotype frequencies generated by fifteen SNPs, we analyzed each pair by simple Chi square tests to avoid errors due to multiple comparisons. The 'A-C-C-C-T-C-C-A-A-T-C-C-C-G-G' haplotype showed statistically significant higher occurrence in the control group compared to DS probands and BC. Frequency of this haplotype was also higher compared to other haplotypes generated from these 15 SNPs, which may be conferring protection towards the diseases.

MDR analysis exhibited high individual entropy value for rs461155 in both BC and OC groups. Involvement of risk allele of rs461155 in subjects with these two solid tumors has also been reported earlier [32]. Therefore, from the present study we predict that rs461155 may individually play an important role in solid tumor groups (BC and OC). On the other hand, rs2070530, rs2070531, rs6517481, rs7276961, rs1051475, rs1051476 and rs11254 may act together in ALL, BC and OC groups, where rs11254 act as a nodal SNP. In silico analysis revealed that, rs 11254 has a potency to change miRNA and TF binding sites in the 3'UTR of ETS2. Presence of risk allele and inappropriate interaction of rs11254 probably can hamper proper expression of ETS2. There are various reports on loss of heterozygosity $(\mathrm{LOH})$ of different genes under different malignant conditions like ovarian tumors [55], BC [56], head and neck squamous cell carcinoma [57], pituitary tumors [58], AML [59] etc. We found 100\% LOH for rs11254 in DS probands.

Analysis of LD pattern of studied SNPs exhibited that rs6517481, rs7276961, rs1051475, rs1051476, rs2070529, rs2070530, rs2070531, rs461155 and rs11254 are in high LD in the studied population. MDR analysis also provided evidence of interaction between these SNPs in the malignant groups and parents of probands with DS and thus, may suggest combined effect of these fSNPs in the studied groups.

Similar to the present observation, SNP pairs rs2070529-rs2070530 were found to be in high LD in other populations studied in the HapMap; LD data for other SNP pairs were not available. Both haplotype distribution pattern and LD between different SNPs were found to vary in different groups examined in the present investigation, which could be attributed to the difference in allelic frequencies. Whether the observed difference is contributing to the disease etiology requires further analysis.

Our results do not imply that ETS2 and SIM2 are the only TFs in the HSA21 with a role in oncogenesis because several other TFs, located in the HSA21, also have association with malignancies [31,60]. For example, increased expression of $\mathrm{BACH} 1$ (transcriptional regulator of megakaryocytic differentiation process) and SON (homologous sequence with MYC family of oncoproteins) were reported in association with myeloid leukemia in DS [61]. RUNX1 and ERG were hypothesized as candidates for leukemia in non-DS patients; however, triplicate 
dosages of these two genes were incapable to generate transient myeloproliferative leukemia in Ts1Cje mice and thus, these two genes may not be directly responsible for development of leukemia in individuals with DS [62]. Further analysis of these TFs, in association with SIM2 and ETS2, would help us to understand their actual role in DS associated malignancies.

\section{Conclusions}

We summarize that, a) the rs2269188 ' $G$ ' allele, showing trend for higher occurrence in ALL patients (BH $\mathrm{P}=0.06$, $\mathrm{OR}=2.6$ ), may play a regulatory role in ALL by altering carcinogen metabolism; in mother of probands with DS also, this SNP may contribute some regulatory role as the individual effect of this SNP calculated by MDR analysis was very high (Table 7); that b) rs711 may have very important role in DS and associated malignancies; that c) the fSNP rs11254 may act as a core SNP in the interaction cluster of rs6517481, rs7276961, rs1051475, rs1051476, rs2070529, rs2070530 and rs2070531, thus playing a role in malignant development in BC, OC, ALL; in parents of DS probands, these SNPs also showed strong interaction while in DS, a high individual effect of rs11254 was found; and that d) rs2070530, rs711 and rs11254 (with 100\% LOH) showed strong genotypic association with DS. This prominent difference in status of ASNPs of SIM2 and ETS2 may indicate a significantly different pattern of SIM2 and ETS2 regulation in the studied groups, eventually leading to altered expression of their downstream genes associated with distinct disease phenotypes.

\section{Additional files}

\section{Additional file 1: Table S1. Sites of overexpression and}

underexpression of SIM2 and ETS2. S2: Possible downstream genes of SIM2 and ETS2 identified by in silico analysis. S3: Details of allelic and genotypic association test of studied SNPS. S4: Comparative analysis of haplotypes in different study group.

\section{Competing interests}

The authors declare that they have no competing interests.

\section{Authors' contributions}

AC: Concept and designing, genotyping, analysis and manuscript preparation. SD: Karyotyping and confirmation of trisomy 21. SM: Collection and DNA isolation of OC samples. NM: Collection and DNA isolation of BC samples. AD: Programming and running of "Consensus-Finder". AM: Recruitment of ALL patients. SS: Recruitment of DS patients. CKP: Supervision of NM's work, consultation during manuscript preparation. KC: Supervision of SM and AD's work, input in manuscript preparation. ALR: Critical evaluation and editing. KM: Concept and design of the work, supervision of whole process, manuscript preparation and final revision. All authors read and approved the final manuscript.

\section{Acknowledgement}

Authors are thankful to all the study participants and the Indian Council of Medical Research for providing senior research fellowship to AC (\#45/1/2010Hum/BMS).

\section{Author details}

'Manovikas Biomedical Research and Diagnostic Centre, MRIH, 482, Madudah, Plot I-24, Sec.-J, E.M. Bypass, Kolkata 700107, India. '2 Indian Institute of Chemical Biology, Kolkata, India. ${ }^{3}$ Chittaranjan National Cancer Institute, Kolkata, India. ${ }^{4}$ Netaji Subhash Chandra Bose Cancer Research Institute, Kolkata, India. ${ }^{5}$ Tufts University School of Medicine, Boston, MA, USA. ${ }^{6}$ Present address: Department of Biochemistry and Molecular Biology, University of Nebraska Medical Center, Nebraska, USA.

Received: 6 February 2012 Accepted: 18 January 2013

Published: 23 January 2013

\section{References}

1. Hasle $H$, Clemmensen $I H$, Mikkelsen M: Risks of leukemia and solid tumors in individuals with Down's syndrome. Lancet 2000, 355:165-169.

2. Chen R, Morgan AA, Dudley J, Deshpande T, Li L, Kodama K, Chiang AP, Butte AJ: FitSNPs: highly differentially expressed genes are more likely to have variants associated with disease. Genome Biol 2008, 9:R170.

3. Michaud J, Fan CM: Single-minded-two genes, three chromosomes. Genome Res 1997, 7:569-571.

4. Yamaki A, Noda S, Kudoh J, Shindoh N, Maeda H, Minoshima S, Kawasaki K, Shimizu Y, Shimizu N: The mammalian single-minded (SIM) gene: mouse cDNA structure and diencephalic expression indicate a candidate gene for Down syndrome. Genomics 1996, 35:136-143.

5. Wharton KA Jr, Franks RG, Kasai Y, Crews ST: Control of CNS midline transcription by asymmetric E-box-like elements: similarity to xenobiotic responsive regulation. Development 1994, 120:3563-3569.

6. Rushmore TH, Kong AN: Pharmacogenomics, regulation and signaling pathways of phase I and II drugmetabolizing enzymes. Curr Drug Metab 2002, 3:481-490.

7. Hankinson O: The aryl hydrocarbon receptor complex. Annu Rev Pharmacol Toxicol 1995, 35:307-340.

8. Nebert DW, Roe AL, Dieter MZ, Solis WA, Yang Y, Dalton TP: Role of the aromatic hydrocarbon receptor and $[\mathrm{Ah}]$ gene battery in the oxidative stress response, cell cycle control, and apoptosis. Biochem Pharmacol 2000, 59:65-85.

9. Young MPD, Tress M, Narayanan R: Identification of Down's syndrome critical locus gene SIM2-s as a drug therapy target for solid tumors. Proc Natl Acad Sci 2003, 100:4760-4765.

10. Aleman MJ, Young MPD: Inhibition of single minded 2 gene expression mediates tumor-selective apoptosis and differentiation in human colon cancer cells. Proc Natl Acad Sci 2005, 102:12765-12770.

11. Halvorsen OJ, Oyan AM, Bo TH, Olsen S, Rostad K, Haukaas SA, Bakke AM, Marzolf B, Dimitrov K, Stordrange L, Lin B, Jonassen I, Hood L, Akslen LA, Kalland $\mathrm{KH}$ : Gene expression profiles in prostate cancer: association with patient subgroups and tumour differentiation. Int J Oncol 2005, 26:329-336.

12. Kwak HI, Gustafson T, Metz RP, Laffin B, Schedin P, Porter WW: Inhibition of breast cancer growth and invasion by single-minded $2 \mathrm{~s}$. Carcinogenesis 2007, 28(2):259-266.

13. Rosa-Rosa JM, Pita G, Urioste M, Llort G, Brunet J, Lázaro C, Blanco I, Ramón Y, Cajal T, Díez O, de la Hoya M, Caldés T, Tejada MI, González-Neira A, Benitez J: Genome-wide linkage scan reveals three putative breastcancer-susceptibility loci. Am J Hum Genet 2009, 84:115-122.

14. Sumarsono SH, Wilson TJ, Tymms MJ, Venter DJ, Corrick CM, Kola R, Lahoud MH, Papas TS, Seth A, Kola I: Down's syndrome-like skeletal abnormalities in Ets2 transgenic mice. Nat London 1996, 379:534-538.

15. Wolvetang EJ, Bradfield OM, Hatzistavrou T, Crack PJ, Busciglio J, Kola I, Hertzog PJ: Overexpression of the chromosome 21 transcription factor Ets2 induces neuronal apoptosis. Neurobiol Dis 2003, 14:349-356.

16. Wolvetang EW, Bradfield OM, Tymms M, Zavarsek S, Hatzistavrou T, Kola I, Hertzog PJ: The chromosome 21 transcription factor ETS2 transactivates the beta-APP promoter: implications for Down syndrome. Biochim Biophys Acta 2003, 1628:105-110.

17. Watson DK, Ascione R, Papas TS: Molecular analysis of the ets genes and their products. Crit Rev Oncog 1990, 1:409-436.

18. Seth A, Ascione R, Fisher RJ, Mavrothalassitis GJ, Bhat NK, Papas TS: The ets gene family. Cell Growth Differ 1992, 3:327-334.

19. Macleod K, Leprince D, Stehelin D: The ets gene family. Trends Biochem Sci 1992, 17:251-256.

20. Wasylyk B, Hahn SL, Giovane A: The Ets family of transcription factors. Eur J Biochem 1993, 211:7-18. 
21. Ghysdael J, Boureux A: The ETS family of Transcriptional regulators. In The ETS family of Transcriptional regulators. Volume 1st edition. Edited by Yaniv M, Ghysdael J. Switzerland: Birkhauser Verlag: Basel; 1997:29-88.

22. Dittmer J, Nordheim A: Ets trsnscription factor and human disease. Biochim Biophys Acta 1998, 1377:F1-F11.

23. Papas TS, Bhat NK, Spyropoulos DD, Mjaatvedt AE, Vournakis J, Seth A, Watson DK: Functional relationships among ETS gene family members. Leukemia 1997, 11(Suppl 3):557-566.

24. Ghosh A, Kolodkin AL: Specification of neuronal connectivity: ETS marks the spot. Cell 1998, 95:303-306.

25. Karim FD, Urness LD, Thummel CS, Klemsz MJ, McKercher SR, Celada A, Van Beveren C, Maki RA, Gunther CV, Nye JA, Graves BJ: The ETSdomain: a new DNA-binding motif that recognizes a purine-rich core DNA sequence. Genes Dev 1990, 4:1451-1453.

26. Sharrocks $A D$, Brown $A L$, Ling $Y$, Yates PR: The ETS-domain transcription factor family. Int J Biochem Cell Biol 1997, 29:1371-1387.

27. Carbone GM, Napoli S, Valentini A, Cavalli F, Watson DK, Catapano CV: Triplex DNA-mediated downregulation of Ets2 expression results in growth inhibition and apoptosis in human prostate cancer cells. Nucl Acids Res 2004, 32:4358-4367.

28. Sementchenko VI, Watson DK: Ets target genes: past, present and future. Oncogene 2000, 19:6533-6548.

29. Baker KM, Wei G, Schaffner AE, Ostrowski MC: Ets-2 and components of mammalian SWI/SNF form a repressor complex that negatively regulates the BRCA1 promoter. J Biol Chem 2003, 278:17876-17884.

30. Papas TS, Watson DK, Sacchi N, Fujiwara S, Seth AK, Fisher RJ, Bhat NK, Mavrothalassitis $\mathrm{G}$, Koizumi $\mathrm{S}$, Jorcyk $\mathrm{CL}$, et al: ETS family of genes in leukemia and Down syndrome. Am J Med Genet 1990, 7:251-261.

31. Stankiewicz MJ, Crispino JD: ETS2 and ERG promote megakaryopoiesis and synergize with alterations in GATA-1 to immortalize hematopoietic progenitor cells. Blood 2009, 113:1347-3337.

32. Chatterjee A, Dutta S, Mukherjee S, Mukherjee N, Chandra S, Mukherjee A, Sinha S, Panda CK, Chaudhuri K, Mukhopadhyay K: Differential allelic distribution of V-ets erythroblastosis virus E26 oncogene homolog2 (ETS2) functional polymorphisms in different group of patients. Gene Express 2011, 15:61-73.

33. Miller SA, Dykes DD, Polesky HF: A simple salting out procedure for extracting DNA from human nucleated cells. Nucl Acid Res 1988, 16:1215.

34. Purcell S, Neale B, Todd-Brown K, Thomas L, Ferreira MAR, Bender D, Maller J, Sklar P, de Bakker PIW, Daly MJ, Sham PC: PLINK: a toolset for whole-genome association and population-based linkage analysis. Am J Hum Genet 2007, 81. http://pngu.mgh.harvard.edu/ purcell/plink/contact.shtml.

35. Dudbridge F: Pedigree disequilibrium tests for multilocus haplotypes. Genet Epidemiol 2003, 25(2):115-121.

36. Mei H, Ma D, Ashley-Koch A, Martin ER: Extension of multifactor dimensionality reduction for identifying multilocus effects in the GAW14 simulated data. BMC Genet 2005, 6:S145-S149.

37. Lenth RV: Statistical power calculations. J Anim Sci 2007, 85:E24-E29.

38. Chatterjee A, Dutta S, Sinha S, Mukhopadhyay K: Exploratory investigation on functional significance of ETS2 and SIM2 genes in Down syndrome. Disease Markers 2011, 31:247-257.

39. Zenger S, He W, Ek-Rylander B, Vassiliou D, Wedin R, Bauer H, Andersson G: Differential expression of tartrate-resistant acid phosphatase isoforms $5 a$ and $5 \mathrm{~b}$ by tumor and stromal cells in human metastatic bone disease. Clin Exp Metastasis 2011, 28(1):65-73.

40. Magklara A, Scorilas A, Katsaros D, Massobrio M, Yousef GM, Fracchioli S, Danese S, Diamandis EP: The human KLK8 (neuropsin/ovasin) gene: identification of Two novel splice variants and its prognostic value in ovarian cancer. Clin Can Res 2001, 7:806-811.

41. Mao R, Wang X, Spitznagel EL Jr, Frelin LP, Ting JC, Ding H, Kim JW Ruczinski I, Downey TJ, Pevsner J: Primary and secondary transcriptional effects in the developing human down syndrome brain and heart. Genome Biol 2005, 6(13):R107.

42. Deutsch S, Lyle R, Dermitzakis ET, Attar H, Subrahmanyan L, Gehrig C, Parand L, Gagnebin M, Rougemont J, Jongeneel CV, Antonarakis SE: Gene expression variation and expression quantitative trait mapping of human chromosome 21 genes. Hum Mol Genet 2005, 14(23):3741-3749.

43. Conti A, Fabbrini F, D'Agostino P, Negri R, Greco D, Genesio R, D'Armiento M Olla C, Paladini D, Zannini M, Nitsch L: Altered expression of mitochondrial and extracellular matrix genes in the heart of human fetuses with chromosome 21 trisomy. BMC Genomics 2007, 8:268.
44. Sommer CA, Pavarino-Bertelli EC, Goloni-Bertollo EM, Henrique-Silva F: Identification of dysregulated genes in lymphocytes from children with down syndrome. Genome 2008, 51:19-29.

45. Lockstone HE, Harris LW, Swatton JE, Wayland MT, Holland AJ, Bahn S: Gene expression proWling in the adult down syndrome brain. Genomics 2007, 90:647-660.

46. Altug-Teber O, Bonin M, Walter M, Mau-Holzmann UA, Dufke A, Stappert H, Tekesin I, Heilbronner H, Nieselt K, Riess O: SpeciWc transcriptional changes in human fetuses with autosomal trisomies. Cytogenet Genome Res 2007, 119:171-184.

47. Patterson D: Molecular genetic analysis of down syndrome. Hum Genet 2009, 126:195-214.

48. Rhodes DR, Yu J, Shankerz K, Deshpande N, Varambally R, Ghosh D, Barrette T, Pandeyband A, Chinnaiyan AM: ONCOMINE: a cancer microarray database and integrated data-mining platform. Neoplasia 2004, 6:1-6. available at: https://www.oncomine.org/resource/login.html.

49. Ross DT, Scherf U, Eisen MB, Perou CM, Rees C, Spellman P, Iyer $V$, Jeffrey SS, de Rijn MV, Waltham M, Pergamenschikov A, Lee JCF, Lashkari D, Shalon D, Myers TG, Weinstein JN, Botstein D, Brown PO: Systematic variation in gene expression patterns in human cancer cell lines. Nat Genet 2000, 24(3):227-234.

50. Mart'ınez A, Olarte I, Mergold MA, Gutierrez M, Rozen E, Collazo J, AmancioChassin O, Ordo'nez RM, Montesinos JJ, Mayani H, McCurdy DK, OstroskyWegman P, Garrido-Guerrero E, Miranda El: mRNA expression of MAGE-A3 gene in leukemia cells. Leukemia Res 2007, 31:33-37.

51. Kuraya M, Matsushita M, Endo Y, Thiel S, Fujita T: Expression of H--ficolin/ Hakata antigen, mannose--binding lectin--associated serine protease (MASP)--1 and MASP--3 by human glioma cell line T98G. Int Immunol 2003, 15(1):109-117.

52. Richards KS, Bommert K, Szabo G, Miles R: Differential expression of $\mathrm{Na}+/ \mathrm{K}+-$ ATPase alpha-subunits in mouse hippocampal interneurones and pyramidal cells. J Physiol 2007, 585(Pt 2):491-505.

53. Zhou ZQ, Cao WH, Xie JJ, Lin J, Shen ZY, Zhang QY, Shen JH, XU LY, Li EM: Expression and prognostic significance of THBS1, Cyr61 and CTGF in esophageal squamous cell carcinoma. BMC Cancer 2009, 9:291.

54. Lee IK, Choi JH, Kim YK, Kim HN, Park KS, Lee JJ, Shin MG, Choi C, Kook H, Hwang TJ: Two single nucleotide polymorphisms of the ETS2 transcriptional factor gene predispose individuals to high-risk acute myelogenous leukemia AML. Blood ASH Annual Meet Abstrac No. 2729 2005, 106.

55. Wang Z-J, Churchman M, Campbell IG, Xu W-H, Yan Z-Y, McCluggage WG, Foulkes WD, Tomlinson IPM: Allele loss and mutation screen at the peutzjeghers (LKB1) locus (19p13.3) In sporadic ovarian tumours. Br J Cancer 1999, 80(1/2):70-72

56. Powierska-Czarny J, Miścicka-Śliwka D, Czarny J, Grzybowski T, Woźniak M, Drewa G, Czechowicz W, Sir J: Analysis of microsatellite instability and loss of heterozygosity in breast cancer with the use of a well characterized multiplex system. Acta Biochimica Polonica 2003, 50:1195-1203.

57. Fan CY, Liu KL, Huang HY, Barnes EL, Swalsky PA, Bakker A, Woods J, Finkelstein SD: Frequent allelic imbalance and loss of protein expression of the DNA repair gene hOGG1 in head and neck squamous cell carcinoma. Lab Investig 2001, 81:1429-1438.

58. Clayton RN, Pfeifer M, Atkinson AB, Belchetz P, Wass JA, Kyrodimou E, Vanderpump M, Simpson D, Bicknell J, Farrell WE: Different patterns of allelic loss (loss of heterozygosity) in recurrent human pituitary tumors provide evidence for multiclonal origins. Clin Cancer Res 2000, 6:3973-3982.

59. Sweetser DA, Chen CS, Blomberg AA, Flowers DA, Galipeau PC, Barrett MT, Heerema NA, Buckley J, Woods WG, Bernstein ID, Reid BJ: Loss of heterozygosity in childhood de novo acute myelogenous leukemia. Blood 2001, 98:1188-1194.

60. Fonatsch C: The role of chromosome 21 in hematology and oncology Genes Chromosomes Cancer 2010, 49:497-508.

61. Zwaan MC, Reinhardt D, Hitzler J, Vyas P: Acute leukemias in children with down syndrome. Pediatr Clin North Am 2008, 55:53-70.

62. Carmichael CL, Majewski IJ, Alexander WS, Metcalf D, Hilton DJ, Hewitt CA, Scott HS: Hematopoietic defects in the Ts1Cje mouse model of down syndrome. Blood 2009, 113:1929-1937.

doi:10.1186/1471-2350-14-12

Cite this article as: Chatterjee et al:: Potential contribution of SIM2 and ETS2 functional polymorphisms in Down syndrome associated malignancies. BMC Medical Genetics 2013 14:12. 